TRANSACTIONS OF THE

AMERICAN MATHEMATICAL SOCIETY

Volume 362, Number 1, January 2010, Pages 117-143

S 0002-9947(09)04561-9

Article electronically published on August 13, 2009

\title{
MIXED BOUNDARY-VALUE PROBLEMS FOR MAXWELL'S EQUATIONS
}

\author{
MARIUS MITREA
}

\begin{abstract}
We study the Maxwell system with mixed boundary conditions in a Lipschitz domain $\Omega$ in $\mathbb{R}^{3}$. It is assumed that two disjoint, relatively open subsets $\Sigma^{e}, \Sigma^{h}$ of $\partial \Omega$ such that $\overline{\Sigma^{e}} \cap \overline{\Sigma^{h}}=\partial \Sigma^{e}=\partial \Sigma^{h}$ have been fixed, and one prescribes the tangential components of the electric and magnetic fields on $\Sigma^{e}$ and $\Sigma^{h}$, respectively. Under suitable geometric assumptions on $\partial \Omega, \Sigma^{e}$ and $\Sigma^{h}$, we prove that this boundary value problem is well-posed when $L^{p_{-}}$ estimates for the nontangential maximal function are sought, with $p$ near 2 . A higher-dimensional version of this result is established as well, in the language of differential forms. This extends earlier work by R. Brown and by the author and collaborators.
\end{abstract}

\section{INTRODUCTION}

Let $\Omega \subset \mathbb{R}^{3}$ be a bounded Lipschitz domain, and fix $k \in \mathbb{C}$ with $\operatorname{Im} k>0$. Under these assumptions, it has been proved in [20, 18 that the boundary problem for the Maxwell system

$$
\left\{\begin{array}{l}
\operatorname{curl} E-i k H=0 \text { in } \Omega, \\
\operatorname{curl} H+i k E=0 \text { in } \Omega, \\
\nu \times\left. E\right|_{\partial \Omega}=f \in L_{t}^{p, \text { Div }}(\partial \Omega), \\
E^{*}, H^{*} \in L^{p}(\partial \Omega)
\end{array}\right.
$$

is well-posed whenever $1<p<2+\varepsilon$, for some small $\varepsilon=\varepsilon(\partial \Omega, k)>0$. Here and elsewhere, $(\cdot)^{*}$ denotes the nontangential maximal function associated with $\Omega$ and all boundary traces are taken in the sense of nontangential limits; see the body of the paper (particularly §2) for all relevant definitions.

The aim of this paper is to continue this study by considering the case when the Maxwell system is equipped with mixed boundary conditions. To state these explicitly, we need some notation. Throughout the paper, we write

$$
\partial \Omega=\Sigma^{e} \bigsqcup \Sigma^{h}
$$

Received by the editors June 21, 2005 and, in revised form, April 12, 2007.

2000 Mathematics Subject Classification. Primary 35J55, 78A30, 42B20; Secondary 35F15, $35 \mathrm{C} 15,78 \mathrm{M} 15$.

Key words and phrases. Maxwell's equations, Lipschitz domains, mixed boundary conditions, Rellich estimates.

The author was supported in part by the NSF and the University of Missouri Office of Research. 
whenever $\Sigma^{e}, \Sigma^{h}$ are two disjoint, relatively open subsets of $\partial \Omega$ such that $\overline{\Sigma^{e}} \cap \overline{\Sigma^{h}}=$ $\partial \Sigma^{e}=\partial \Sigma^{h}$. Having fixed two such sets, $\Sigma^{e}, \Sigma^{h}$, we then prescribe

$$
\nu \times\left. E\right|_{\Sigma^{e}}=f \text { on } \Sigma^{e} \text { and } \nu \times\left. H\right|_{\Sigma^{h}}=g \text { on } \Sigma^{h} .
$$

This type of boundary condition is physically relevant since it arises naturally in semi-conductor modeling [13, 14, 15], where $\Sigma^{e}$ is the insulating part and $\Sigma^{h}$ represents the electric contacts. The boundary conditions (1.3) also appear naturally in many microwave problems; cf. the discussion in [1]. In [9], necessary and sufficient conditions for the solvability of magnetostatic and electrostatic problems with mixed boundary conditions in Lipschitz domains are presented. Another context where mixed boundary conditions intervene naturally is scattering phenomena from partially coated perfect conductors. Cf. [5] and the survey [4].

At the scalar level, R. Brown 3 was the first to succeed in treating the case of the Helmholtz operator with $L^{2}$ mixed (Dirichlet and Neumann) boundary conditions:

$$
\left\{\begin{array}{l}
\left(\Delta+k^{2}\right) u=0 \text { in } \Omega, \\
\left.\nabla_{\tan } u\right|_{\Sigma_{D}} \text { prescribed in } L^{2}\left(\Sigma_{D}\right), \\
\left.\partial_{\nu} u\right|_{\Sigma_{N}} \text { prescribed in } L^{2}\left(\Sigma_{N}\right)
\end{array}\right.
$$

in a suitable subclass of Lipschitz domains, referred to in the sequel as creased domains. Informally speaking, this requires the two pieces $\Sigma_{D}$ and $\Sigma_{N}$, in which $\partial \Omega$ is partitioned, to meet at an angle $<\pi$. Brown also made the observation that this type of restriction is necessary given that the harmonic function $u(x, y)=$ $\operatorname{Im}(x+i y)^{1 / 2}, x \in \mathbb{R}, y>0$, has zero tangential and normal derivatives on the positive and negative real semi-axis, respectively. This analysis has been subsequently extended to allow $L^{p}$-data with $1<p \leq 2$ in $[22$.

Here we take the first steps aimed at generalizing this work from scalar equations to systems. Even though, in broad outline, we follow the same approach as in [3], the technical details are rather different, as the algebra is considerably more subtle. We now recall the class of creased domains introduced by R. Brown in [3] (specialized to the three-dimensional setting). Let $\Omega \subseteq \mathbb{R}^{3}$ be a bounded Lipschitz domain. Assume that $\partial \Omega$ can be decomposed into two pieces $\Sigma^{e}, \Sigma^{h}$ as in (1.2) and that there exist two numbers $\kappa_{e} \geq 0, \kappa_{h} \geq 0$ with $\kappa_{e}+\kappa_{h}>0$, which also satisfy the following properties. For each boundary point $x_{o}$ there exist $r>0$ and two Lipschitz functions $\varphi: \mathbb{R}^{2} \rightarrow \mathbb{R}$ and $\psi: \mathbb{R} \rightarrow \mathbb{R}$ for which

$$
\begin{aligned}
& \Omega \cap B_{2 r}\left(x_{o}\right)=B_{2 r}\left(x_{o}\right) \cap\left\{\left(x_{1}, x_{2}, x_{3}\right): x_{3}>\varphi\left(x_{1}, x_{2}\right)\right\}, \\
& \Sigma^{e} \cap B_{2 r}\left(x_{o}\right)=B_{2 r}\left(x_{o}\right) \cap \partial \Omega \cap\left\{\left(x_{1}, x_{2}, x_{3}\right): x_{1}<\psi\left(x_{2}\right)\right\}, \\
& \Sigma^{h} \cap B_{2 r}\left(x_{o}\right)=B_{2 r}\left(x_{o}\right) \cap \partial \Omega \cap\left\{\left(x_{1}, x_{2}, x_{3}\right): x_{1}>\psi\left(x_{2}\right)\right\},
\end{aligned}
$$

and such that

$$
\begin{aligned}
& \partial_{x_{1}} \varphi \geq \kappa_{e} \text { on }\left\{\left(x_{1}, x_{2}\right): x_{1}<\psi\left(x_{2}\right)\right\}, \\
& \partial_{x_{1}} \varphi \leq-\kappa_{h} \text { on }\left\{\left(x_{1}, x_{2}\right): x_{1}>\psi\left(x_{2}\right)\right\} .
\end{aligned}
$$

Roughly speaking, the two pieces $\Sigma^{e}$ and $\Sigma^{h}$ are separated by a Lipschitz curve, $\Gamma:=\partial \Sigma^{e}=\partial \Sigma^{h}$ (the 'crease' of the domain) and meet at an angle which is strictly 


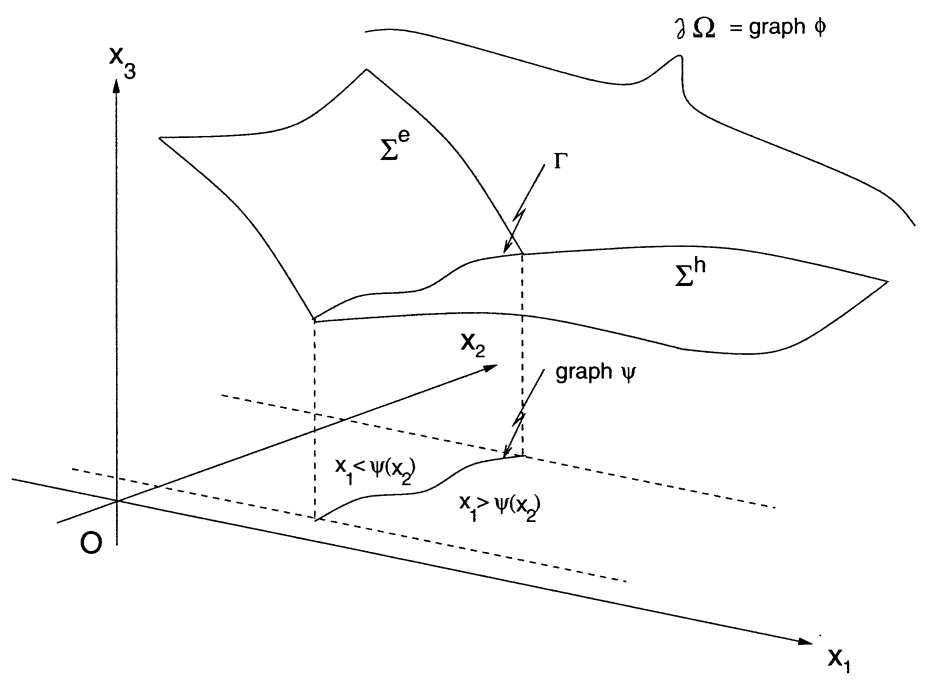

FIGURE 1

less than $\pi$. This is illustrated in Figure 1. Thus, $\partial \Omega$ is inherently non-smooth. As pointed out by Brown 3 the class of creased domains is natural, since in the class of domains with piece-wise boundaries the well-posedness of (1.4) fails precisely when (1.6) is violated.

Our first main result is as follows.

Theorem 1.1. Let $\Omega, \Sigma^{e}, \Sigma^{h}$ be as above, and fix $k \in \mathbb{C}$ with $\operatorname{Im} k>0$. Then there exists a small $\varepsilon=\varepsilon\left(\partial \Omega, \Sigma^{e}, \Sigma^{h}, k\right)>0$ and a finite $C=C\left(\partial \Omega, \Sigma^{e}, \Sigma^{h}, k\right)>0$ with the following significance.

Assume that $p \in(2-\varepsilon, 2+\varepsilon)$ and let $f \in L_{t}^{p \text {,Div }}\left(\Sigma^{e}\right)$ and $g \in L_{t}^{p \text {,Div }}\left(\Sigma^{h}\right)$ be arbitrary. Then the Maxwell system with mixed boundary conditions,

$$
\left\{\begin{array}{l}
\operatorname{curl} E-i k H=0 \text { in } \Omega, \\
\operatorname{curl} H+i k E=0 \text { in } \Omega, \\
\nu \times\left. E\right|_{\Sigma^{e}}=f \text { on } \Sigma^{e}, \\
\nu \times\left. H\right|_{\Sigma^{h}}=g \text { on } \Sigma^{h}, \\
E^{*}, H^{*} \in L^{p}(\partial \Omega),
\end{array}\right.
$$

has a a unique solution. Moreover, this solution satisfies the natural estimate

$$
\left\|E^{*}\right\|_{L^{p}(\partial \Omega)}+\left\|H^{*}\right\|_{L^{p}(\partial \Omega)} \leq C\|f\|_{L_{t}^{p, \text { Div }}\left(\Sigma^{e}\right)}+C\|g\|_{L_{t}^{p, \text { Div }}\left(\Sigma^{h}\right)} \text {. }
$$

A few remarks are in order here. First, the condition that $f \in L_{t}^{p, \text { Div }}\left(\Sigma^{e}\right)$, $g \in L_{t}^{p \text {,Div }}\left(\Sigma^{h}\right)$ is also necessary given the class of functions in which a solution $(E, H)$ is sought. This amounts to asking $f, g$ to be tangential fields, with $L^{p}$ integrable components, and such that their surface divergence is in $L^{p}$ as well. Second, when $k \in \mathbb{R}$, the problem (1.7) is, generally speaking, only Fredholm solvable. Third, while our current methods allow us to treat the case when $|p-2|$ is small, we conjecture that the optimal range of $p$ 's is $1<p<2+\varepsilon$. 
There is a higher-dimensional, higher-degree extension of this result. To state it, call a bounded Lipschitz domain $\Omega$ in $\mathbb{R}^{n}, n \geq 2$, a creased domain, in the sense of Brown, if (1.5)-(1.6) hold with $x_{3}$ replaced by $x_{n}$ and $x_{2}$ replaced by $x^{\prime \prime}:=\left(x_{2}, \ldots, x_{n-1}\right)$ (with a natural interpretation when $\left.n=2\right)$. In this setting, if $k \in \mathbb{C}, \operatorname{Im} k \geq 0$ and $\ell \in\{0,1,2, \ldots, n\}$, the goal is to determine two differential forms $E$ and $H$, respectively, of degrees $(\ell+1)$ and $\ell$, in $\Omega$ such that

$$
\left\{\begin{array}{l}
\delta E-i k H=0 \text { in } \Omega, \\
d H+i k E=0 \text { in } \Omega, \\
E^{*}, H^{*} \in L^{p}(\partial \Omega), \\
\left.\nu \vee E\right|_{\Sigma^{e}}=f \in L_{t}^{p, \delta}\left(\Sigma^{e}, \Lambda^{\ell}\right), \\
\left.\nu \wedge H\right|_{\Sigma^{h}}=g \in L_{n}^{p, d}\left(\Sigma^{h}, \Lambda^{\ell+1}\right) .
\end{array}\right.
$$

Theorem 1.2. With the above notation and hypotheses, there exists $\varepsilon=$ $\varepsilon\left(\partial \Omega, \Sigma^{e}, \Sigma^{h}, k\right)>0$ such that (1.9) is well-posed whenever $p \in(2-\varepsilon, 2+\varepsilon)$.

Let us point out that this last result contains both Theorem 1.1, to which it reduces when $n=3$, after some simple, natural identifications, and Brown's main result in 3], pertaining to the well-posedness of (1.4). The latter is obtained by taking $p=2, \ell=0, \Sigma^{e}=\Sigma_{N}, \Sigma^{h}=\Sigma_{D}$, and then setting $u:=H$. In particular, the class of creased domains retains its significance in the present context as well.

Even though Theorem 1.2 is our most general result, we opted to first present a detailed proof for Theorem 1.1, and then highlight both the differences and the analogies between this and its more involved higher-dimensional/degree version. The plan of the paper is as follows. In $\S 2$ we review potential theoretic concepts, first in the three-dimensional context (for vector fields; cf. §2.1) and then in Euclidean spaces of arbitrary dimension (working with differential forms; cf. §2.2). Certain new Rellich type estimates, most useful in the context of (1.7), are deduced in $\S 3$. Then, in $\S 4$, the graph-domain version of (1.7) is proved to be well-posed for purely imaginary wave numbers. This argument is subsequently adapted to the bounded domain case (and $k \in \mathbb{C}$ with $\operatorname{Im} k>0$ ) in $\S 5$, where the proof of Theorem 1.1 is completed. Finally, in $\S 6$, we discuss the proof of Theorem 1.2

In closing, we would like to point out that the structural richness of the higherdegree case (cf. [19, 2]) allows for a larger variety of mixed problems which can be treated along similar lines. In turn, such results are useful for proving regularity results for differential forms with mixed boundary conditions. A typical result reads as follows:

$$
u, d u, \delta u \in L^{2}(\Omega) \text { and }\left.\nu \wedge u\right|_{\Sigma_{n}} \in L^{2}\left(\Sigma_{n}\right),\left.\nu \vee u\right|_{\Sigma_{t}} \in L^{2}\left(\Sigma_{t}\right) \Rightarrow u \in H^{1 / 2,2}(\Omega)
$$

granted that $\Omega$ is a creased domain with boundary splitting $\partial \Omega=\Sigma_{n} \sqcup \Sigma_{t}$. We plan to return to this topic on a different occasion.

\section{Preliminaries}

2.1. Vector potential theory. Call a bounded domain $\Omega \subset \mathbb{R}^{3}$ Lipschitz if the following condition is satisfied. For each boundary point $x \in \partial \Omega$ there exist a neighborhood $U$ of $x$ in $\mathbb{R}^{3}$, a system of coordinates (isometric to the standard one) in $\mathbb{R}^{3}$ and a real-valued Lipschitz function $\varphi$ such that $\partial \Omega \cap U=U \cap \operatorname{graph} \varphi$, where the graph of $\varphi$ is considered in the new system of coordinates. 
Let $d \sigma$ be the surface measure on $\partial \Omega$ so that $\nu$, the outward unit normal to $\Omega$, is well-defined $d \sigma$-a.e. on $\partial \Omega$. Also, for some sufficiently large, fixed, $\mu>0$ let $\gamma(x):=\{y \in \Omega:|x-y| \leq(1+\mu)$ dist $(y, \partial \Omega)\}$ be a nontangential cone with vertex at $x \in \partial \Omega$. The nontangential boundary trace of a (possibly vector-valued) function $u$ defined in $\Omega$ is then defined as

$$
\left.u\right|_{\partial \Omega}(x):=\lim _{\substack{z \rightarrow x \\ z \in \gamma(x)}} u(z) \text { for } x \in \partial \Omega .
$$

Also, the nontangential maximal function of $u$ is defined by

$$
u^{*}(x):=\sup \{|u(z)|: z \in \gamma(x)\} \quad \text { for } \quad x \in \partial \Omega .
$$

Next, we briefly discuss layer potential operators associated with the Helmholtz operator $\Delta+k^{2}$ and the Lipschitz domain $\Omega \subset \mathbb{R}^{3}$. Recall that for each $k \in \mathbb{C}$,

$$
\Phi_{k}(x):=-\frac{e^{i k|x|}}{4 \pi|x|}, \quad x \in \mathbb{R}^{3} \backslash\{0\},
$$

is the canonical (radial) fundamental solution for $\Delta+k^{2}$ in $\mathbb{R}^{3}$. The single layer acoustic potential operator and its boundary versions are then defined by

$$
\begin{aligned}
& \mathcal{S}_{k} f(x):=\int_{\partial \Omega} \Phi_{k}(x-y) f(y) d \sigma(y), \quad x \in \Omega, \\
& S_{k} f(x):=\int_{\partial \Omega} \Phi_{k}(x-y) f(y) d \sigma(y), \quad x \in \partial \Omega .
\end{aligned}
$$

The action of the operators $\mathcal{S}_{k}, S_{k}$ on vector fields is defined component-wise. It is then easy to check that

$$
\left.\mathcal{S}_{k} f\right|_{\partial \Omega}(x)=S_{k} f(x), \quad x \in \partial \Omega .
$$

In addition, at almost any $x \in \partial \Omega$,

$$
\left.\partial_{\nu} \mathcal{S}_{k} f\right|_{\partial \Omega}=\left(-\frac{1}{2} I+K_{k}^{*}\right) f
$$

where $\partial_{\nu}=\sum_{j} \nu_{j} \partial_{j}$ is the normal derivative and $K_{k}^{*}$ is the formal transpose of the principal-value integral operator

$$
K_{k} f(x):=\text { p.v. } \frac{1}{4 \pi} \int_{\partial \Omega} \frac{\langle\nu(y), y-x\rangle}{|y-x|^{3}} e^{i k|y-x|}(1-i k|y-x|) f(y) d \sigma(y), x \in \partial \Omega,
$$

the so-called (boundary-version) double layer acoustic potential operator. Combining the techniques of $[8]$ with the results in [6] we can infer that for any vector field $f$ in $L^{p}\left(\partial \Omega, \mathbb{R}^{3}\right), 1<p<\infty$, at almost any $x \in \partial \Omega$ we have

$$
\left.\operatorname{div} \mathcal{S}_{k} f\right|_{\partial \Omega}(x)=-\frac{1}{2}\langle\nu(x), f(x)\rangle+\text { p.v. } \int_{\partial \Omega} \operatorname{div}_{x}\left\{\Phi_{k}(x-y) f(y)\right\} d \sigma(y)
$$

and

$$
\left.\operatorname{curl} \mathcal{S}_{k} f\right|_{\partial \Omega}(x)=-\frac{1}{2} \nu(x) \times f(x)+\text { p.v. } \int_{\partial \Omega} \operatorname{curl}_{x}\left\{\Phi_{k}(x-y) f(y)\right\} d \sigma(y) .
$$

Turning to function spaces, we next set

$$
L_{t}^{p}(\partial \Omega):=\left\{f \in L^{p}\left(\partial \Omega, \mathbb{R}^{3}\right):\langle\nu, f\rangle=0 \text { a.e. on } \partial \Omega\right\} .
$$


From the above discussion and since for $f \in L_{t}^{p}(\partial \Omega)$ one has $\nu \times(\nu \times f)=-f$, we see that

$$
\nu \times\left.\operatorname{curl} \mathcal{S}_{k} f\right|_{\partial \Omega}=\left(\frac{1}{2} I+M_{k}\right) f, \quad \text { a.e. on } \partial \Omega,
$$

where, for a vector-valued density $f$ on $\partial \Omega$,

$$
M_{k} f(x):=\nu(x) \times\left[\text { p.v. } \int_{\partial \Omega} \operatorname{curl}_{x}\left\{\Phi_{k}(x-y) f(y)\right\} d \sigma(y)\right], \quad x \in \partial \Omega,
$$

is the so-called magnetic dipole operator on $\partial \Omega$. Once again relying on the results of [6] one can show that, for each $1<p<\infty$, the operator $M_{k}$ is a bounded mapping of $L_{t}^{p}(\partial \Omega)$.

For a scalar function $\eta$, set $\nabla_{\tan } \eta:=\nabla \eta-\left(\partial_{\nu} \eta\right) \nu$ for the tangential gradient and, for $1<p<\infty$, define the Sobolev space $L_{1}^{p}(\partial \Omega)$ as the collection of all $\eta \in L^{p}(\partial \Omega)$ with $\nabla_{\tan } \eta \in L_{t}^{p}(\partial \Omega)$. Furthermore, set $L_{-1}^{p}(\partial \Omega):=\left(L_{1}^{p^{\prime}}(\partial \Omega)\right)^{*}, 1 / p+1 / p^{\prime}=1$. Now, if $1<p<\infty$ and $f \in L_{t}^{p}(\partial \Omega)$, we define $\operatorname{Div} f$ as the functional

$$
\langle\operatorname{Div} f, \eta\rangle:=-\int_{\partial \Omega}\left\langle f, \nabla_{\tan } \eta\right\rangle d \sigma,
$$

where $\eta$ is an arbitrary Lipschitz continuous function on $\partial \Omega$. Thus, Div is welldefined as a mapping from $L_{t}^{p}(\partial \Omega)$ into $L_{-1}^{p}(\partial \Omega)$ and

$$
\operatorname{Div}(\nu \times f)=-\langle\nu, \operatorname{curl} f\rangle
$$

for any vector field $f \in L^{p}\left(\partial \Omega, \mathbb{R}^{3}\right)$. Note that $\nu \times f \in L_{t}^{p}(\partial \Omega)$ and that the operator $\langle\nu, \operatorname{curl} \cdot\rangle$ involves only tangential derivatives so that the right-hand side of (2.14) is well-defined and belongs to $L_{-1}^{p}(\partial \Omega)$.

If $\Omega=\left\{x_{3}>\varphi\left(x^{\prime}\right): x^{\prime}=\left(x_{1}, x_{2}\right) \in \mathbb{R}^{2}\right\}$, then the vector fields

$$
T^{1}\left(x^{\prime}, \varphi\left(x^{\prime}\right)\right):=\frac{\left(1,0, \partial_{1} \varphi\left(x^{\prime}\right)\right)}{\sqrt{1+\left|\nabla \varphi\left(x^{\prime}\right)\right|^{2}}}, \quad T^{2}\left(x^{\prime}, \varphi\left(x^{\prime}\right)\right):=\frac{\left(0,1, \partial_{2} \varphi\left(x^{\prime}\right)\right)}{\sqrt{1+\left|\nabla \varphi\left(x^{\prime}\right)\right|^{2}}}
$$

form a basis for the tangent plane to $\partial \Omega$ at the point $\left(x^{\prime}, \varphi\left(x^{\prime}\right)\right)$ for almost every $x^{\prime} \in \mathbb{R}^{2}$.

Proposition 2.1. For each $1<p<\infty$, the following are true:

(i) A vector field $f: \partial \Omega \rightarrow \mathbb{R}^{3}$ belongs to $L_{t}^{p}(\partial \Omega)$ if and only if there exist scalar functions $a_{1}, a_{2} \in L^{p}(\partial \Omega)$ such that $f=a_{1} T^{1}+a_{2} T^{2}$ on $\partial \Omega$. Furthermore,

$$
\|f\|_{L^{p}\left(\partial \Omega, \mathbb{R}^{3}\right)} \approx\left\|a_{1}\right\|_{L^{p}(\partial \Omega)}+\left\|a_{2}\right\|_{L^{p}(\partial \Omega)},
$$

where the intervening constants depend only on $\|\nabla \varphi\|_{L^{\infty}}$.

(ii) A vector field $f=a_{1} T^{1}+a_{2} T^{2}, a_{1}, a_{2} \in L^{p}(\partial \Omega)$, belongs to $L_{t}^{p \text {,Div }}(\partial \Omega)$ if and only

$$
\frac{\partial a_{1}}{\partial T^{1}}+\frac{\partial a_{2}}{\partial T^{2}} \in L^{p}(\partial \Omega)
$$

In this case,

$$
\operatorname{Div} f=\frac{\partial a_{1}}{\partial T^{1}}+\frac{\partial a_{2}}{\partial T^{2}}
$$

so that, in particular,

$$
\|\operatorname{Div} f\|_{L^{p}(\partial \Omega)} \approx\left\|\operatorname{div}_{\mathbb{R}^{2}}\left(a_{1}\left(x^{\prime}, \varphi\left(x^{\prime}\right)\right), a_{2}\left(x^{\prime}, \varphi\left(x^{\prime}\right)\right)\right)\right\|_{L^{p}\left(\mathbb{R}^{2}\right)} .
$$

Once again, the proportionality constants depend only on $\|\nabla \varphi\|_{L^{\infty}}$. 
Proof. The crux of the matter is the observation that, if $f=a_{1} T^{1}+a_{2} T^{2}, a_{1}, a_{2}$ scalars on $\partial \Omega$, then for any $\eta$ we have

$$
\int_{\partial \Omega}\left\langle f, \nabla_{\tan } \eta\right\rangle d \sigma=\sum_{j=1,2} \int_{\partial \Omega} a_{j} \frac{\partial \eta}{\partial T^{j}} d \sigma=-\int_{\partial \Omega}\left(\sum_{j=1,2} \frac{\partial a_{j}}{\partial T^{j}}\right) \eta d \sigma
$$

after an integration by parts.

Next we study the action of the surface divergence operator in connection with the boundary integral operators introduced earlier. Two identities are going to be important for us here. First, for $k \in \mathbb{C}, 1<p<\infty$ and $f \in L_{t}^{p}(\partial \Omega)$, we have

$$
\operatorname{div}\left(\mathcal{S}_{k} f\right)=\mathcal{S}_{k}(\operatorname{Div} f) \quad \text { in } \Omega .
$$

Second, if $f \in L_{t}^{p}(\partial \Omega)$, then

$$
\operatorname{Div}\left(M_{k} f\right)=-k^{2}\left\langle\nu, S_{k} f\right\rangle-K_{k}^{*}(\operatorname{Div} f) \quad \text { in } \quad L_{-1}^{p}(\partial \Omega) .
$$

In particular, $M_{k}$ is well-defined and bounded on the space

$$
L_{t}^{p, \text { Div }}(\partial \Omega):=\left\{f \in L_{t}^{p}(\partial \Omega): \operatorname{Div} f \in L^{p}(\partial \Omega)\right\},
$$

equipped with the natural norm, for each $1<p<\infty$. Moreover, it has been proved in [20], 18] that there exists $\varepsilon=\varepsilon(\partial \Omega)>0$ such that the operators $\pm \frac{1}{2} I+M_{k}$ : $L_{t}^{p \text {,Div }}(\partial \Omega) \rightarrow L_{t}^{p \text {,Div }}(\partial \Omega)$ are isomorphisms if $1<p<2+\varepsilon$ and $\operatorname{Im} k>0$.

Finally, we specialize the wave number $k$ by taking $k \in i \mathbb{R}_{+}$, i.e. $k=i \omega, \omega>0$. Then for each $1<p<\infty$,

$$
\begin{aligned}
& \omega\left\|\left(\mathcal{S}_{i \omega} f\right)^{*}\right\|_{L^{p}(\partial \Omega)}+\left\|\left(\nabla \mathcal{S}_{i \omega} f\right)^{*}\right\|_{L^{p}(\partial \Omega)} \leq C\|f\|_{L^{p}(\partial \Omega)}, \\
& \omega\left\|S_{i \omega} f\right\|_{L^{p}(\partial \Omega)}+\left\|K_{i \omega} f\right\|_{L^{p}(\partial \Omega)}+\left\|K_{i \omega}^{*} f\right\|_{L^{p}(\partial \Omega)} \leq C\|f\|_{L^{p}(\partial \Omega)},
\end{aligned}
$$

where $C=C(\partial \Omega, p)>0$ is independent of $\omega>0$. It is essentially well known (cf. 77 for the case $\omega=0$ ) that for each $1<p<2+\varepsilon$ the operators $\pm \frac{1}{2} I+K_{i \omega}$ are isomorphisms of $L^{p}(\partial \Omega)$. This will play a role in $\S 4$.

For homogeneity reasons, we find it convenient to equip the space (2.23) with a family of (mutually equivalent) norms indexed by $\omega>0$ :

$$
\|f\|_{L_{t}^{p, \operatorname{Div}}(\partial \Omega), \omega}:=\|f\|_{L^{p}\left(\partial \Omega, \mathbb{R}^{3}\right)}+\omega^{-1}\|\operatorname{Div} f\|_{L^{p}(\partial \Omega)} .
$$

It follows that for each $p \in(1, \infty)$ there exists $C=C(\partial \Omega, p)>0$, independent of $\omega$, such that

$$
\left\|M_{i \omega} f\right\|_{L_{t}^{p, \operatorname{Div}}(\partial \Omega), \omega} \leq C\|f\|_{L_{t}^{p, \text { Div }}(\partial \Omega), \omega}
$$

uniformly in $\omega>0$. In fact, from the work in [20, 18] we infer that there exists $\varepsilon=\varepsilon(\partial \Omega)>0$ such that for each $1<p<2+\varepsilon$ the operators $\pm \frac{1}{2} I+M_{i \omega}$ are isomorphisms of $L_{t}^{p \text {,Div }}(\partial \Omega)$ and, granted that $2-\varepsilon<p<2+\varepsilon$,

$$
\left\|\left( \pm \frac{1}{2} I+M_{i \omega}\right) f\right\|_{L_{t}^{p, \text { Div }}(\partial \Omega), \omega} \approx\|f\|_{L_{t}^{p, \text { Div }}(\partial \Omega), \omega}
$$

uniformly in $\omega>0$. 
2.2. Differential forms rudiments. We start by reviewing some basic definitions; cf. 23] for more details. For $0 \leq \ell \leq n$, we let $\Lambda^{\ell}:=\Lambda^{\ell} \mathbb{R}^{n}$ stand for the vector space of differential forms of degree $\ell$ with real (or complex) coefficients. That is, $E \in \Lambda^{\ell}$ if and only if $E=\sum_{|I|=\ell}^{\prime} E_{I} d x^{I}$, where the sum is performed over all increasing multi-indices $I \in\{1,2, \ldots, n\}^{\ell}$ and $d x^{I}$ stands for $d x_{i_{1}} \wedge \cdots \wedge d x_{i_{\ell}}$ if $I=\left(i_{1}, i_{2}, \ldots, i_{\ell}\right)$. For $E, F \in \Lambda^{\ell}$ set $\langle E, F\rangle:=\sum_{|I|=\ell}^{\prime} E_{I} F_{I}, \bar{E}:=\sum_{I}^{\prime} \bar{E}_{I} d x^{I}$, and $|E|:=\langle E, \bar{E}\rangle^{1 / 2}$. Recall that the Hodge $*$-operator, $*: \Lambda^{\ell} \mathbb{R}^{n} \rightarrow \Lambda^{n-\ell} \mathbb{R}^{n}$, is defined by

$$
* E:=\sum_{|I|=\ell}^{\prime} \varepsilon_{\{1, \ldots, n\}}^{I I^{c}} E_{I} d x^{I^{c}},
$$

where $I^{c}:=\{1,2, \ldots, m\} \backslash I$ and, for any two ordered arrays $I, J$,

$$
\varepsilon_{J}^{I}:=\left\{\begin{array}{l}
0, \text { if } I \text { and } J \text { do not coincide as sets, } \\
\text { the sign of the permutation taking } I \text { onto } J, \text { otherwise. }
\end{array}\right.
$$

As is well known, $* *=(-1)^{\ell(n-\ell)}$ on $\Lambda^{\ell}$. In the sequel $\wedge$ and $\vee$ will denote, respectively, the exterior and interior products of forms. For example, if $\alpha=$ $\left(\alpha_{1}, \alpha_{2}, \ldots, \alpha_{n}\right)$ is a vector field, identified with the 1-form $\alpha=\alpha_{1} d x_{1}+\cdots+\alpha_{n} d x_{n}$, and if $E=\sum_{|I|=\ell}^{\prime} E_{I} d x^{I} \in \Lambda^{\ell}$, then

$$
\alpha \wedge E=\sum_{|J|=\ell+1} \prime\left(\sum_{|I|=\ell}^{\prime} \sum_{i} \varepsilon_{J}^{i I} \alpha_{i} E_{I}\right) d x^{J} \quad \text { and } \quad \alpha \vee E=\sum_{|J|=\ell-1}{ }^{\prime}\left(\sum_{i} \alpha_{i} E_{i J}\right) d x^{J} .
$$

Some elementary properties of these operators are collected below.

Lemma 2.2. For $\alpha, \beta \in \mathbb{R}^{n}$ and $E, F \in \Lambda^{\ell}, G \in \Lambda^{\ell-1}$, the following are true:

(1) $\alpha \wedge(\alpha \wedge E)=0$ and $\alpha \vee(\alpha \vee E)=0$;

(2) $*(\alpha \wedge E)=(-1)^{\ell} \alpha \vee * E$ and $*(\alpha \vee E)=(-1)^{\ell+1} \alpha \wedge * E$;

(3) $\alpha \wedge(\beta \vee E)+\beta \vee(\alpha \wedge E)=\langle\alpha, \beta\rangle E$;

(4) $\langle\alpha \vee E, G\rangle=\langle E, \alpha \wedge G\rangle$.

We recall that the classical exterior differential operator $d, d:=\sum_{i=1}^{n} \partial_{i} d x_{i}$, acts on a form $E=\sum_{I}^{\prime} E_{I} d x^{I}$ by

$$
d E:=\sum_{I}^{\prime} \sum_{i} \partial_{i} E_{I} d x_{i} \wedge d x^{I}
$$

Its formal transpose $\delta$, the so called co-differential operator, is then given by

$$
\delta E:=-\sum_{|J|=\ell-1}^{\prime} \sum_{i} \partial_{i} E_{i J} d x^{J}
$$

with the convention that, if $I$ is a permutation of an increasing multi-index $\bar{I}$, then we set $E_{I}:=\varepsilon_{\bar{I}}^{I} E_{\bar{I}}$.

Lemma 2.3. Assume that $\Omega$ is a bounded domain in $\mathbb{R}^{n}$ with a sufficiently smooth boundary. Denote by $\nu$ and $d \sigma$ the unit normal and the surface area on $\partial \Omega$, respectively. Then the following hold:

(1) $d^{2}=0, \delta^{2}=0$ and $-(d \delta+\delta d)=\Delta$, the Laplacian in $\mathbb{R}^{n}$;

(2) $\delta=(-1)^{n(\ell+1)+1} * d *$ and $* \delta=(-1)^{\ell} d *, \delta *=(-1)^{\ell+1} * d$ on $\ell$-forms; 
(3) For $E \in C^{1}\left(\bar{\Omega}, \Lambda^{\ell}\right)$ and $F \in C^{1}\left(\bar{\Omega}, \Lambda^{\ell+1}\right)$,

$$
\int_{\Omega}\langle d E, F\rangle d x=\int_{\Omega}\langle E, \delta F\rangle d x+\int_{\partial \Omega}\langle\nu \wedge E, F\rangle d \sigma .
$$

Furthermore, if $E \in C^{2}\left(\bar{\Omega}, \Lambda^{\ell}\right)$ has $\left(\Delta+k^{2}\right) E=0$ on $\Omega$, then

$$
\int_{\Omega}\left\{|d E|^{2}+|\delta E|^{2}-k^{2}|E|^{2}\right\} d x=\int_{\partial \Omega}\{\langle d E, \nu \wedge \bar{E}\rangle-\langle\delta E, \nu \vee \bar{E}\rangle\} d \sigma .
$$

We now introduce a distinguished elementary solution $\Phi_{k}$ for the Helmholtz operator $\Delta+k^{2}$ in $\mathbb{R}^{n}, k \in \mathbb{C}_{+}$, and $n \geq 1$. Concretely, at each $x \in \mathbb{R}^{n} \backslash\{0\}$, set

$$
\Phi_{k}(x):=\left\{\begin{array}{l}
-\frac{1}{\omega_{n}} \frac{(-i k)^{n-2}}{(n-2) !} \int_{1}^{\infty}\left(t^{2}-1\right)^{\frac{n-3}{2}} e^{i k t|x|} d t, \text { for } n \geq 2, \\
\frac{e^{i k|x|}}{2 i k}, \text { for } n=1
\end{array}\right.
$$

where $\omega_{n}$ denotes the area of the unit sphere in $\mathbb{R}^{n}$. See [10] for more details. Note that (2.35) reduces precisely to (2.3) when $n=3$.

Now let $\Omega$ be a Lipschitz domain in $\mathbb{R}^{n}$ and, in analogy to (2.4), define the single layer acoustic potential operator on differential forms $f \in L^{p}\left(\partial \Omega, \Lambda^{\ell}\right):=$ $L^{p}(\partial \Omega) \otimes \Lambda^{\ell}, 1<p<\infty$, by

$$
\mathcal{S}_{k} f(x):=\int_{\partial \Omega} \Phi_{k}(x-y) f(y) d \sigma(y), \quad x \in \Omega .
$$

From [6] and classical arguments, we infer the following.

Lemma 2.4. Let $\Omega$ be a Lipschitz domain in $\mathbb{R}^{n}$, and let $f \in L^{p}\left(\partial \Omega, \Lambda^{\ell}\right), 0 \leq \ell \leq n$, $1<p<\infty$. Then, at almost every point $x \in \partial \Omega$,

$$
\begin{gathered}
\lim _{\substack{z \rightarrow x \\
z \in \gamma(x)}} \mathcal{S}_{k} f(z)=S_{k} f(x):=\int_{\partial \Omega} \Phi_{k}(x-y) f(y) d \sigma(y), \\
\lim _{\substack{z \rightarrow x \\
z \in \gamma(x)}} d \mathcal{S}_{k} f(z)=-\frac{1}{2}(\nu \wedge f)(x)+\text { p.v. }\left(d \int_{\partial \Omega} \Phi_{k}(x-y) f(y) d \sigma(y)\right), \\
\lim _{\substack{z \rightarrow x^{x} \\
z \in \gamma(x)}} \delta \mathcal{S}_{k} f(z)=\frac{1}{2}(\nu \vee f)(x)+\text { p.v. }\left(\delta \int_{\partial \Omega} \Phi_{k}(x-y) f(y) d \sigma(y)\right) .
\end{gathered}
$$

Moreover, there exists $C=C(\partial \Omega, p)>0$ such that

$(2.40) \omega\left\|\left(\mathcal{S}_{i \omega} f\right)^{*}\right\|_{L^{p}(\partial \Omega)}+\left\|\left(d \mathcal{S}_{i \omega} f\right)^{*}\right\|_{L^{p}(\partial \Omega)}+\left\|\left(\delta \mathcal{S}_{i \omega} f\right)^{*}\right\|_{L^{p}(\partial \Omega)} \leq C\|f\|_{L^{p}\left(\partial \Omega, \Lambda^{\ell}\right)}$, uniformly in $\omega>0$.

We continue to assume that $\Omega$ is a Lipschitz domain in $\mathbb{R}^{n}$. A differential form $E$ defined a.e. on $\partial \Omega$ is called tangential if $\nu \vee E=0$ a.e. on $\partial \Omega$, and normal if $\nu \wedge E=0$ a.e. on $\partial \Omega$. The tangential component of $E, E_{t}$, is given by $E_{t}:=$ $\nu \vee(\nu \wedge E)$, whereas the normal component is given by $E_{n}:=\nu \wedge(\nu \vee E)$. This terminology is suggested by the fact that $E=E_{t}+E_{n}$ and $\left\langle E_{t}, E_{n}\right\rangle=0$, so that $|E|^{2}=\left|E_{t}\right|^{2}+\left|E_{n}\right|^{2}$. Also, note that $\left|E_{t}\right|=|\nu \wedge E|$ and $\left|E_{n}\right|=|\nu \vee E|$. We let $L_{t}^{p}\left(\partial \Omega, \Lambda^{\ell}\right)$ denote the vector space of tangential $\ell$-forms with coefficients from $L^{p}(\partial \Omega)$, and let $L_{n}^{p}\left(\partial \Omega, \Lambda^{\ell}\right)$ stand for the vector space of normal $\ell$-forms with coefficients from $L^{p}(\partial \Omega)$. 
Next, define the boundary exterior derivative of a tangential differential form $f \in L_{l o c}^{1}\left(\partial \Omega, \Lambda^{\ell}\right)$ as the functional

$$
\left\langle\delta_{\partial} f, \psi\right\rangle:=\int_{\partial \Omega}\langle d \psi, f\rangle d \sigma, \quad \forall \psi \in C^{1}\left(\mathbb{R}^{n}, \Lambda^{\ell-1}\right),
$$

and then introduce

$$
L_{t}^{p, \delta}\left(\partial \Omega, \Lambda^{\ell}\right):=\left\{f \in L_{t}^{p}\left(\partial \Omega, \Lambda^{\ell}\right): \delta_{\partial} f \in L^{p}\left(\partial \Omega, \Lambda^{\ell-1}\right)\right\},
$$

equipped with the family of mutually equivalent norms

$$
\|f\|_{L_{t}^{p, \delta}\left(\partial \Omega, \Lambda^{\ell}\right), \omega}:=\|f\|_{L^{p}\left(\partial \Omega, \Lambda^{\ell}\right)}+\omega^{-1}\left\|\delta_{\partial} f\right\|_{L^{p}\left(\partial \Omega, \Lambda^{\ell-1}\right)}, \quad \omega>0 .
$$

Similarly, define the boundary exterior co-derivative of a differential form $f \in$ $L_{l o c}^{1}\left(\partial \Omega, \Lambda^{\ell}\right)$, normal to $\partial \Omega$, as the functional

$$
\left\langle d_{\partial} f, \psi\right\rangle:=\int_{\partial \Omega}\langle\delta \psi, f\rangle d \sigma, \quad \forall \psi \in C^{1}\left(\mathbb{R}^{n}, \Lambda^{\ell+1}\right),
$$

and then introduce

$$
L_{n}^{p, d}\left(\partial \Omega, \Lambda^{\ell}\right):=\left\{f \in L_{n}^{p}\left(\partial \Omega, \Lambda^{\ell}\right): d_{\partial} f \in L^{p}\left(\partial \Omega, \Lambda^{\ell+1}\right)\right\},
$$

equipped with the family of mutually equivalent norms

$$
\|f\|_{L_{n}^{p, d}\left(\partial \Omega, \Lambda^{\ell}\right), \omega}:=\|f\|_{L^{p}\left(\partial \Omega, \Lambda^{\ell}\right)}+\omega^{-1}\left\|d_{\partial} f\right\|_{L^{p}\left(\partial \Omega, \Lambda^{\ell+1}\right)}, \quad \omega>0 .
$$

Clearly, for each $0 \leq \ell \leq n$, the Hodge star-operator is an isomorphism between $L_{n}^{p, d}\left(\partial \Omega, \Lambda^{\ell}\right)$ and $L_{t}^{p, \delta}\left(\partial \Omega, \Lambda^{n-\ell}\right)$. Another useful consequence of definitions is the following.

Lemma 2.5. Let $\Omega$ be a Lipschitz domain in $\mathbb{R}^{n}$. If $E \in C^{1}\left(\Omega, \Lambda^{\ell}\right)$ is such that $E^{*},(\delta E)^{*} \in L^{p}(\partial \Omega)$ for some $1<p<\infty$, and such that $E, \delta E$ have nontangential boundary traces on $\partial \Omega$, then

$$
\delta_{\partial}(\nu \vee E)=-\nu \vee \delta E .
$$

Analogously, if $E^{*},(d E)^{*} \in L^{p}(\partial \Omega)$ and $E, d E$ have nontangential boundary traces on $\partial \Omega$, then

$$
d_{\partial}(\nu \wedge E)=-\nu \wedge d E .
$$

Next we define two principal-value integral operators acting on a differential form $f$ on $\partial \Omega$ by

$$
\begin{aligned}
& M_{k} f(x):=\lim _{\varepsilon \rightarrow 0}\left(\nu(x) \vee d \int_{|x-y| \geq \varepsilon} \Phi_{k}(x-y) f(y) d \sigma(y)\right), \quad x \in \partial \Omega, \\
& N_{k} f(x):=\lim _{\varepsilon \rightarrow 0}\left(\nu(x) \wedge \delta \int_{|y-y| \geq \varepsilon} \Phi_{k}(x-y) f(y) d \sigma(y)\right), \quad x \in \partial \Omega .
\end{aligned}
$$

Note that $M_{k} *=-* N_{k}$. It follows from the results of [6] and well-known techniques that, for each $1<p<\infty$ and each $0 \leq l \leq n$, the operator $M_{k}$ is well-defined and bounded on $L_{t}^{p}\left(\partial \Omega, \Lambda^{\ell}\right)$. Moreover, since

$$
\delta_{\partial} M_{k} f=-k^{2} \nu \vee S_{k} f+\nu \vee d S_{k}\left(\delta_{\partial} f\right), \quad \forall f \in L_{t}^{p, \delta}\left(\partial \Omega, \Lambda^{\ell}\right),
$$

we may also conclude that $M_{k}$ is well-defined and bounded on $L_{t}^{p, \delta}\left(\partial \Omega, \Lambda^{\ell}\right)$. Similarly, the operator $N_{k}$ is well-defined and bounded on $L_{n}^{p}\left(\partial \Omega, \Lambda^{\ell}\right)$ and $L_{n}^{p, d}\left(\partial \Omega, \Lambda^{\ell}\right)$. Moreover, an identity analogous in spirit to (2.50) holds for this operator as well. 
As a final note, we would like to mention here that invertibility results for the operator $\pm \frac{1}{2} I+M_{k}, \pm \frac{1}{2} I+N_{k}$ on these spaces (with $p$ near 2) have been established in [12] and [18].

\section{Rellich TYPE ESTIMATES FOR VECTOR FIELDS}

Assume that $\Omega$ is an arbitrary Lipschitz domain in $\mathbb{R}^{3}$ and denote by $\nu$ its outward unit normal and by $d \sigma$ the surface measure on $\partial \Omega$. Next consider a vector field with real-valued components $\Theta \in C^{1} \cap L^{\infty}$ in $\mathbb{R}^{3}$ so that $\nabla \Theta \in L^{\infty}$. Also, let $U \in C^{2}\left(\Omega, \mathbb{C}^{3}\right)$ be such that $\Delta U \in L^{2}(\Omega),(\nabla U)^{*} \in L^{2}(\partial \Omega)$, and so that the nontangential trace $\left.(\nabla U)\right|_{\partial \Omega}$ exists in $L^{2}(\partial \Omega)$. Under these conditions, the following Rellich type identity can be verified via successive integrations by parts:

$$
\begin{aligned}
& \frac{1}{2} \int_{\partial \Omega}\left\{|\nu \times \operatorname{curl} U|^{2}\langle\nu, \Theta\rangle-|\langle\nu, \operatorname{curl} U\rangle|^{2}\langle\nu, \Theta\rangle-|\operatorname{div} U|^{2}\langle\nu, \Theta\rangle\right\} d \sigma \\
& \quad+\operatorname{Re} \int_{\partial \Omega}\{\langle\nu \times \operatorname{curl} \bar{U}, \Theta\rangle \operatorname{div} U-\langle\nu \times \operatorname{curl} \bar{U}, \nu \times \Theta\rangle\langle\nu, \operatorname{curl} U\rangle\} d \sigma \\
& =\int_{\Omega}\left\{\frac{1}{2}|\operatorname{curl} U|^{2} \operatorname{div} \Theta+\frac{1}{2}|\operatorname{div} U|^{2} \operatorname{div} \Theta-\operatorname{Re}\langle\operatorname{curl} \bar{U},(\nabla \Theta) \operatorname{curl} U\rangle\right\} d x \\
& \quad-\operatorname{Re} \int_{\Omega}\langle\operatorname{curl} \bar{U}, \operatorname{curl} \Theta\rangle(\operatorname{div} U) d x-\operatorname{Re} \int_{\Omega}\langle\Theta \times \operatorname{curl} \bar{U}+(\operatorname{div} \bar{U}) \Theta, \Delta U\rangle d x .
\end{aligned}
$$

See [20], 18] for related matters. We now specialize this identity to the following situation. Fix $\omega>0$ and let $E, H$ solve

$$
\left\{\begin{array}{l}
\operatorname{curl} E=-\omega H \text { in } \Omega, \\
\operatorname{curl} H=\omega E \text { in } \Omega,
\end{array}\right.
$$

i.e., Maxwell's system with wave number $k=i \omega$, and assume that $E^{*}, H^{*} \in L^{2}(\partial \Omega)$ and the boundary restrictions $\left.E\right|_{\partial \Omega},\left.H\right|_{\partial \Omega}$ exist a.e. in the nontangential limit sense. Furthermore, decompose the boundary $\partial \Omega=\Sigma^{e} \sqcup \Sigma^{h}$ as in (1.2), and assume that it is possible to find a constant vector field $\Theta$ such that

$$
\langle\nu, \Theta\rangle \leq-\kappa \text { a.e. on } \Sigma^{e} \text { and }\langle\nu, \Theta\rangle \geq \kappa \text { a.e. on } \Sigma^{h},
$$

for some fixed $\kappa>0$. Taking $U:=H$ in $\Omega$ we may then write, based on (3.1),

$$
\begin{aligned}
\int_{\Sigma^{e}} & {\left[-\frac{1}{2}\langle\nu, \Theta\rangle\right]|\langle\nu, E\rangle|^{2} d \sigma+\int_{\Sigma^{h}} \frac{1}{2}\langle\nu, \Theta\rangle|\nu \times E|^{2} d \sigma } \\
= & \int_{\Sigma^{h}} \frac{1}{2}\langle\nu, \Theta\rangle|\langle\nu, E\rangle|^{2} d \sigma-\int_{\Sigma^{e}} \frac{1}{2}\langle\nu, \Theta\rangle|\nu \times E|^{2} d \sigma \\
& \quad+\int_{\partial \Omega} \mathcal{O}(|\langle\nu, E\rangle||\nu \times E|) d \sigma+\omega \int_{\Omega} \mathcal{O}(|E||H|) d x .
\end{aligned}
$$

In turn, these allow us to estimate

$$
\begin{aligned}
& \int_{\Sigma^{e}}|\langle\nu, E\rangle|^{2} d \sigma+\int_{\Sigma^{h}}|\nu \times E|^{2} d \sigma \\
& \leq C \int_{\Sigma^{h}}|\langle\nu, E\rangle|^{2} d \sigma+C \int_{\Sigma^{e}}|\nu \times E|^{2} d \sigma+C \omega \int_{\Omega}\left\{|E|^{2}+|H|^{2}\right\} d x .
\end{aligned}
$$


In order to continue, observe that

$$
\begin{aligned}
\int_{\partial \Omega}\langle\nu \times E, H\rangle d \sigma & =\int_{\Omega}\langle\operatorname{curl} E, H\rangle d x-\int_{\Omega}\langle E, \operatorname{curl} H\rangle d x \\
& =-\omega \int_{\Omega}\left\{|E|^{2}+|H|^{2}\right\} d x .
\end{aligned}
$$

Consequently, using this, (1.2), and Cauchy-Schwarz's inequality, we see that for each $\varepsilon>0$ there exists $C_{\varepsilon}>0$ such that

$$
\begin{gathered}
\omega \int_{\Omega}\left\{|E|^{2}+|H|^{2}\right\} d x \leq \\
C_{\varepsilon} \int_{\Sigma^{e}}|\nu \times E|^{2} d \sigma+C_{\varepsilon} \int_{\Sigma^{h}}|\nu \times H|^{2} d \sigma \\
+\varepsilon \int_{\partial \Omega}\left\{|E|^{2}+|H|^{2}\right\} d \sigma .
\end{gathered}
$$

Hence, the Pythagorean Theorem, (1.2), (3.5) and (3.7), yield

$$
\begin{aligned}
\int_{\partial \Omega}|E|^{2} d \sigma & =\int_{\partial \Omega}|\langle\nu, E\rangle|^{2} d \sigma+\int_{\partial \Omega}|\nu \times E|^{2} d \sigma \\
& \leq C_{\varepsilon} \int_{\Sigma^{h}}|\langle\nu, E\rangle|^{2} d \sigma+C_{\varepsilon} \int_{\Sigma^{e}}|\nu \times E|^{2} d \sigma+\varepsilon \int_{\partial \Omega}\left\{|E|^{2}+|H|^{2}\right\} d \sigma,
\end{aligned}
$$

for each $\varepsilon>0$. In fact, a similar reasoning in which we reverse the roles of $E$ and $H$, on the one hand, and the roles of $\Sigma^{e}$ and $\Sigma^{h}$, on the other hand, gives

$$
\int_{\partial \Omega}|H|^{2} d \sigma \leq C_{\varepsilon} \int_{\Sigma^{e}}|\langle\nu, H\rangle|^{2} d \sigma+C_{\varepsilon} \int_{\Sigma^{h}}|\nu \times H|^{2} d \sigma+\varepsilon \int_{\partial \Omega}\left\{|E|^{2}+|H|^{2}\right\} d \sigma
$$

for each $\varepsilon>0$. Adding up (3.8), (3.9), absorbing the terms with small coefficients in the left-hand side, and keeping in mind that

$$
\operatorname{Div}(\nu \times E)=\omega\langle\nu, H\rangle, \quad \operatorname{Div}(\nu \times H)=-\omega\langle\nu, E\rangle,
$$

finally leads to the conclusion that

$$
\begin{aligned}
\int_{\partial \Omega}\left\{|E|^{2}+|H|^{2}\right\} d \sigma \leq & C \int_{\Sigma^{e}}\left\{|\nu \times E|^{2}+\omega^{-2}|\operatorname{Div}(\nu \times E)|^{2}\right\} d \sigma \\
& +C \int_{\Sigma^{h}}\left\{|\nu \times H|^{2}+\omega^{-2}|\operatorname{Div}(\nu \times H)|^{2}\right\} d \sigma .
\end{aligned}
$$

For further reference we also note that

$$
\begin{aligned}
\omega \int_{\Omega}\left\{|E|^{2}+|H|^{2}\right\} d x \leq & C \int_{\Sigma^{e}}\left\{|\nu \times E|^{2}+\omega^{-2}|\operatorname{Div}(\nu \times E)|^{2}\right\} d \sigma \\
& +C \int_{\Sigma^{h}}\left\{|\nu \times H|^{2}+\omega^{-2}|\operatorname{Div}(\nu \times H)|^{2}\right\} d \sigma,
\end{aligned}
$$

thanks to (3.6) and (3.11).

\section{Well-POSEDNESS IN THE GRAPH CASE}

Let $\Omega \subset \mathbb{R}^{3}$ be a Lipschitz domain. For an open subset $\Sigma \subseteq \partial \Omega$ and $1<p<\infty$ set

$$
\begin{aligned}
& L_{t}^{p}(\Sigma):=\left\{f \in L^{p}\left(\Sigma, \mathbb{R}^{3}\right):\langle\nu, f\rangle=0 \text { a.e. on } \Sigma\right\} \\
& L_{t}^{p, \text { Div }}(\Sigma):=\left\{\left.f\right|_{\Sigma}: f \in L_{t}^{p, \text { Div }}(\partial \Omega)\right\} \\
& L_{t, 0}^{p, \text { Div }}(\Sigma):=\left\{f \in L_{t}^{p, \text { Div }}(\Sigma): \tilde{f} \in L_{t}^{p, \text { Div }}(\partial \Omega)\right\}
\end{aligned}
$$


where the tilde denotes the extension by zero to $\partial \Omega$. Much as with (2.23)-(2.25), we equip the second space listed above with a family of (mutually equivalent) norms indexed by $\omega>0$ :

$$
\|f\|_{L_{t}^{p, \operatorname{Div}}(\Sigma), \omega}:=\|f\|_{L^{p}\left(\Sigma, \mathbb{R}^{3}\right)}+\omega^{-1}\|\operatorname{Div} f\|_{L^{p}(\Sigma)} .
$$

A similar family of norms is used in conjunction with $L_{t, 0}^{p \text {,Div }}(\Sigma)$. More specifically, we set

$$
\|f\|_{L_{t, 0}^{p, \operatorname{Div}}(\Sigma), \omega}:=\|\tilde{f}\|_{L_{t}^{p, \operatorname{Div}}(\Omega), \omega}:=\|\tilde{f}\|_{L^{p}\left(\Omega, \mathbb{R}^{3}\right)}+\omega^{-1}\|\operatorname{Div} \tilde{f}\|_{L^{p}(\Sigma)} .
$$

More can be said when $\Sigma$ is "reasonable" (which is going to be the case for the applications we have in mind). Concretely, let $\pi: \mathbb{R}^{3} \rightarrow \mathbb{R}^{2}, \pi(x):=x^{\prime}$ be the natural projection onto $\mathbb{R}^{2}$ and assume that $\Sigma \subset \partial \Omega$ is such that $\pi \Sigma$, the image of $\Sigma$ under $\pi$, is a Lipschitz domain in $\mathbb{R}^{2}$. In this situation, it follows from Proposition 2.1, [17] and a dilation argument that

$$
\|f\|_{L_{t}^{p, \text { Div }}(\Sigma), \omega} \approx \inf \left\{\|F\|_{L^{p}\left(\Sigma, \mathbb{R}^{3}\right)}+\omega^{-1}\|\operatorname{Div} F\|_{L^{p}(\Sigma)}: F \in L_{t}^{p, \operatorname{Div}}(\partial \Omega),\left.F\right|_{\Sigma}=f\right\}
$$

with constants which depend only on the Lipschitz character of $\Omega$ and $\pi \Sigma$.

Let $\Omega \subseteq \mathbb{R}^{3}$ be the domain above the graph of the Lipschitz function $\varphi: \mathbb{R}^{2} \rightarrow \mathbb{R}$. Assume that there exist yet another Lipschitz function $\psi: \mathbb{R} \rightarrow \mathbb{R}$ and constants

$$
\kappa_{e} \geq 0, \quad \kappa_{h} \geq 0 \text { with } \kappa_{e}+\kappa_{h}>0
$$

such that

$$
\begin{aligned}
& \partial_{x_{1}} \varphi \geq \kappa_{e} \text { on }\left\{\left(x_{1}, x_{2}\right): x_{1}<\psi\left(x_{2}\right)\right\} \\
& \partial_{x_{1}} \varphi \leq-\kappa_{h} \text { on }\left\{\left(x_{1}, x_{2}\right): x_{1}>\psi\left(x_{2}\right)\right\} .
\end{aligned}
$$

We set

$$
\begin{aligned}
& \Sigma^{e}:=\partial \Omega \cap\left\{\left(x_{1}, x_{2}, x_{3}\right): x_{1}<\varphi\left(x_{2}\right)\right\}, \\
& \Sigma^{h}:=\partial \Omega \cap\left\{\left(x_{1}, x_{2}, x_{3}\right): x_{1}>\varphi\left(x_{2}\right)\right\},
\end{aligned}
$$

and observe that if we take the vector field $\Theta:=\left(1,0,\left(\kappa_{e}-\kappa_{h}\right) / 2\right)$, then the estimates in (3.3) hold with $\kappa:=\frac{1}{2}\left(\kappa_{e}+\kappa_{h}\right)\left(1+\|\nabla \varphi\|_{L^{\infty}}\right)^{-1 / 2}>0$.

Our goal is to prove the well-posedness of (1.7) in this graph setting for $k \in i \mathbb{R}_{+}$, say $k=i \omega, \omega>0$. To this end, fix $\omega>0$ and let $\varepsilon=\varepsilon(\partial \Omega)>0$ be sufficiently small. It has been shown in [20, 18] that, if $1<p<2+\varepsilon$, then for each $f \in L_{t, 0}^{p \text {, Div }}\left(\Sigma^{e}\right)$ the boundary problem

$$
\left\{\begin{array}{l}
\operatorname{curl} E=-\omega H \text { in } \Omega, \\
\operatorname{curl} H=\omega E \text { in } \Omega, \\
\nu \times H=\tilde{f} \text { on } \partial \Omega, \\
E^{*}, H^{*} \in L^{p}(\partial \Omega)
\end{array}\right.
$$

is well-posed. Therefore, the operator

$$
\Lambda: L_{t, 0}^{p, \text { Div }}\left(\Sigma^{e}\right) \longrightarrow L_{t}^{p, \text { Div }}\left(\Sigma^{e}\right), \quad \Lambda f:=\left.(\nu \times E)\right|_{\Sigma^{e}}
$$


is well-defined, linear and bounded. Thus, matters are reduced to showing that $\Lambda$ is an isomorphism if $2-\varepsilon<p<2+\varepsilon$. We proceed in a series of steps, starting with

Step I. We claim that the operator in question is bounded from below when $p=2$ (assuming that the norms (4.2), (4.3) are used). Indeed, thanks to the Rellich estimate (3.11) we may write

$$
\begin{aligned}
\|f\|_{L_{t, 0}^{2, \text { Div }}\left(\Sigma^{e}\right), \omega}^{2} \approx & \int_{\partial \Omega}\left\{|\tilde{f}|^{2}+\omega^{-2}|\operatorname{Div} \tilde{f}|^{2}\right\} d \sigma \\
= & \int_{\partial \Omega}\left\{|\nu \times H|^{2}+\omega^{-2}|\operatorname{Div}(\nu \times H)|^{2}\right\} d \sigma \\
= & \int_{\partial \Omega}\left\{|\nu \times H|^{2}+|\langle\nu, E\rangle|^{2}\right\} d \sigma \\
\leq & \int_{\partial \Omega}\left\{|E|^{2}+|H|^{2}\right\} d \sigma \\
\leq & C \int_{\Sigma^{e}}\left\{|\nu \times E|^{2}+\omega^{-2}|\operatorname{Div}(\nu \times E)|^{2}\right\} d \sigma \\
& +C \int_{\Sigma^{h}}\left\{|\nu \times H|^{2}+\omega^{-2}|\operatorname{Div}(\nu \times H)|^{2}\right\} d \sigma \\
= & C\|\Lambda f\|_{L_{t}^{2, \text { Div }}\left(\Sigma^{e}\right), \omega}^{2}
\end{aligned}
$$

where the last step utilizes the fact that $\nu \times H=0$ on $\Sigma^{h}$. As always, the intervening constants depend exclusively on $\|\nabla \varphi\|_{L^{\infty}}$. This aspect is going to play an important role later on.

Step II. We now construct a one-parameter family of operators $\left\{\Lambda_{\theta}\right\}_{\theta}$ indexed continuously for $\theta \in[0,1]$ and such that $\Lambda=\Lambda_{1}$. Concretely, following [3] we introduce

$$
\varphi_{0}\left(x^{\prime}\right):=\kappa \max \left\{x_{1}, 2 \psi\left(x_{2}\right)-x_{1}\right\}, \quad \varphi_{\theta}:=\theta \varphi+(1-\theta) \varphi_{0}, \theta \in[0,1],
$$

and set

$$
\begin{aligned}
& \Omega_{\theta}:=\left\{x_{3}>\varphi_{\theta}\left(x^{\prime}\right): x^{\prime} \in \mathbb{R}^{2}\right\}, \\
& \Sigma_{\theta}^{e}:=\partial \Omega_{\theta} \cap\left\{x \in \mathbb{R}^{3}: x_{1}>\psi\left(x_{2}\right)\right\}, \\
& \Sigma_{\theta}^{h}:=\partial \Omega_{\theta} \cap\left\{x \in \mathbb{R}^{3}: x_{1}<\psi\left(x_{2}\right)\right\} .
\end{aligned}
$$

For each $\theta \in[0,1]$, consider now the lifting operator $L_{\theta}$ defined as the linear isomorphism

$$
L^{p}\left(\mathbb{R}^{2}, \mathbb{R}^{2}\right) \ni\left(a_{1}, a_{2}\right) \stackrel{L_{\theta}}{\longmapsto} a_{1}(\pi(\cdot)) T_{\theta}^{1}+a_{2}(\pi(\cdot)) T_{\theta}^{2} \in L_{t}^{p}\left(\partial \Omega_{\theta}\right),
$$

where $T_{\theta}^{j}, j=1,2$, are as in (2.15) but with $\varphi_{\theta}$ in place of $\varphi$. Then $L_{\theta}$ can also naturally be viewed as an isomorphism mapping

$$
L_{\theta}: L_{t}^{p, \text { Div }}\left(\pi \Sigma^{e}\right) \longrightarrow L_{t}^{p, \text { Div }}\left(\Sigma_{\theta}^{e}\right) \text { and } L_{\theta}: L_{t, 0}^{p, \text { Div }}\left(\pi \Sigma^{e}\right) \longrightarrow L_{t, 0}^{p, \text { Div }}\left(\Sigma_{\theta}^{e}\right) .
$$


Let $\nu_{\theta}$ stand for the outward unit normal to $\partial \Omega_{\theta}$. A straightforward computation yields

$$
\begin{gathered}
\forall f=\left(f_{1}, f_{2}, f_{3}\right) \in L^{p}\left(\partial \Omega_{\theta}, \mathbb{R}^{3}\right) \Longrightarrow\left(L_{\theta}\right)^{-1}\left(\nu_{\theta} \times f\right)\left(x^{\prime}\right) \\
=\left(f_{2}\left(x^{\prime}, \varphi_{\theta}\left(x^{\prime}\right)\right)-\left(\partial_{2} \varphi_{\theta}\right)\left(x^{\prime}\right) f_{3}\left(x^{\prime}, \varphi_{\theta}\left(x^{\prime}\right)\right),\right. \\
\left.\quad-f_{1}\left(x^{\prime}, \varphi_{\theta}\left(x^{\prime}\right)\right)-\left(\partial_{1} \varphi_{\theta}\right)\left(x^{\prime}\right) f_{3}\left(x^{\prime}, \varphi_{\theta}\left(x^{\prime}\right)\right)\right) .
\end{gathered}
$$

It is also convenient to lift scalar-valued functions via

$$
L_{\theta}: L^{p}\left(\mathbb{R}^{2}\right) \longrightarrow L^{p}\left(\partial \Omega_{\theta}\right), \quad L_{\theta}(f):=f(\pi(\cdot)) .
$$

Once again, this operator naturally adapts as an isomorphism between

$$
L_{\theta}: L^{p}\left(\pi \Sigma^{e}\right) \longrightarrow L^{p}\left(\pi \Sigma_{\theta}^{e}\right) \text { and } L_{\theta}: L_{1}^{p}\left(\pi \Sigma^{e}\right) \longrightarrow L_{1}^{p}\left(\pi \Sigma_{\theta}^{e}\right) .
$$

In what follows, no notational distinction will be made between (4.13), (4.14), (4.16), and (4.17), as the nature of the operator should, in each particular case, be clear from the context.

If $\Lambda_{\theta}$ stands for the analogue of (4.9) constructed in connection with $\Omega_{\theta}$ and $\Sigma_{\theta}^{e}$ in place of $\Omega$ and $\Sigma^{e}$, respectively, then the composition

$$
\bar{\Lambda}_{\theta}: L_{t, 0}^{p, \text { Div }}\left(\pi \Sigma^{e}\right) \longrightarrow L_{t}^{p, \text { Div }}\left(\pi \Sigma^{e}\right), \quad \bar{\Lambda}_{\theta}:=\left(L_{\theta}\right)^{-1} \circ \Lambda_{\theta} \circ L_{\theta}
$$

is well-defined, linear and bounded. The claim that we make at this stage is that

$$
[0,1] \ni \theta \longmapsto \bar{\Lambda}_{\theta} \in \mathcal{L}\left(L_{t, 0}^{p, \text { Div }}\left(\pi \Sigma^{e}\right), L_{t}^{p, \text { Div }}\left(\pi \Sigma^{e}\right)\right)
$$

is continuous.

As a first step in this direction we recall from [20, 18, that the solution $(E, H)$ of (4.8) can be expressed in terms of the data $\tilde{f}$ as

$$
\begin{aligned}
H & =\operatorname{curl} \mathcal{S}_{i \omega}\left[\left(\frac{1}{2} I+M_{i \omega}\right)^{-1} \tilde{f}\right] \\
E & =\omega^{-1} \operatorname{curl} H=\left\{(-\Delta+\nabla \operatorname{div}) \mathcal{S}_{i \omega}\left[\left(\frac{1}{2} I+M_{i \omega}\right)^{-1} \tilde{f}\right]\right\} \\
& =-\omega \mathcal{S}_{i \omega}\left[\left(\frac{1}{2} I+M_{i \omega}\right)^{-1} \tilde{f}\right]+\omega^{-1} \nabla \mathcal{S}_{i \omega}\left[\operatorname{Div}\left(\frac{1}{2} I+M_{i \omega}\right)^{-1} \tilde{f}\right],
\end{aligned}
$$

where it is useful to keep in mind that curl curl $=-\Delta+\nabla$ div. Next, note from (2.22) that

$\operatorname{Div}\left[\left(\frac{1}{2} I+M_{i \omega}\right)^{-1} \tilde{f}\right]=\left(\frac{1}{2} I-K_{i \omega}^{*}\right)^{-1}[\operatorname{Div} \tilde{f}]-\omega^{2}\left(\frac{1}{2} I-K_{i \omega}^{*}\right)^{-1}\left(\left\langle\nu, S_{i \omega} \tilde{f}\right\rangle\right)$,

so that

$$
\begin{aligned}
E= & -\omega \mathcal{S}_{i \omega}\left[\left(\frac{1}{2} I+M_{i \omega}\right)^{-1} \tilde{f}\right]+\omega^{-1} \nabla \mathcal{S}_{i \omega}\left[\left(\frac{1}{2} I-K_{i \omega}^{*}\right)^{-1}(\operatorname{Div} \tilde{f})\right] \\
& -\omega \nabla \mathcal{S}_{i \omega}\left[\left(\frac{1}{2} I-K_{i \omega}^{*}\right)^{-1}\left(\left\langle\nu, S_{i \omega} \tilde{f}\right\rangle\right)\right] .
\end{aligned}
$$


If we now label with the subscript $\theta$ objects defined in connection with $\partial \Omega_{\theta}$ much as they have been originally introduced for $\partial \Omega$, we see that the analogue of the operator (4.9) becomes

$$
\begin{aligned}
\Lambda_{\theta} f= & -\omega \nu_{\theta} \times\left. S_{i \omega, \theta}\left[\left(\frac{1}{2} I+M_{i \omega, \theta}\right)^{-1} \tilde{f}\right]\right|_{\Sigma_{\theta}^{e}} \\
& +\left.\omega^{-1}\left(\nu_{\theta} \times \nabla\right) S_{i \omega, \theta}\left[\left(\frac{1}{2} I-K_{i \omega, \theta}^{*}\right)^{-1}(\operatorname{Div} \tilde{f})\right]\right|_{\Sigma_{\theta}^{e}} \\
& -\left.\omega\left(\nu_{\theta} \times \nabla\right) S_{i \omega, \theta}\left[\left(\frac{1}{2} I-K_{i \omega, \theta}^{*}\right)^{-1}\left(\left\langle\nu_{\theta}, S_{i \omega, \theta} \tilde{f}\right\rangle\right)\right]\right|_{\Sigma_{\theta}^{e}}
\end{aligned}
$$

for each $f \in L_{t, 0}^{p, \operatorname{Div}}\left(\Sigma_{\theta}^{e}\right)$. Let us also note that, thanks to (2.14),

$$
\operatorname{Div}\left(\Lambda_{\theta} f\right)=-\left.\omega\left\langle\nu_{\theta}, \operatorname{curl} S_{i \omega, \theta}\left[\left(\frac{1}{2} I+M_{i \omega, \theta}\right)^{-1} \tilde{f}\right]\right\rangle\right|_{\Sigma_{\theta}^{e}}
$$

whenever $f \in L_{t, 0}^{p \text {, Div }}\left(\Sigma_{\theta}^{e}\right)$. Consequently, the fact that the mapping (4.19) is continuous will follow as soon as we can prove similar continuity claims for the assignments $\theta \mapsto \Psi_{j}(\theta), 1 \leq j \leq 6$, where

$$
\begin{aligned}
\Psi_{1}(\theta):= & \left(L_{\theta}\right)^{-1}\left(\nu_{\theta} \times \omega S_{i \omega, \theta}\right) L_{\theta} \in \mathcal{L}\left(L^{p}\left(\mathbb{R}^{2}, \mathbb{R}^{2}\right), L^{p}\left(\mathbb{R}^{2}, \mathbb{R}^{2}\right)\right), \\
\Psi_{2}(\theta):= & \left(L_{\theta}\right)^{-1}\left(\frac{1}{2} I+M_{i \omega, \theta}\right)^{-1} L_{\theta}=\left[\left(L_{\theta}\right)^{-1}\left(\frac{1}{2} I+M_{i \omega, \theta}\right) L_{\theta}\right]^{-1} \\
& \in \mathcal{L}\left(L^{p}\left(\mathbb{R}^{2}, \mathbb{R}^{2}\right), L^{p}\left(\mathbb{R}^{2}, \mathbb{R}^{2}\right)\right), \\
\Psi_{3}(\theta):= & \left(L_{\theta}\right)^{-1}\left[\left(\nu_{\theta} \times \nabla\right) S_{i \omega, \theta}\right] L_{\theta} \in \mathcal{L}\left(L^{p}\left(\mathbb{R}^{2}\right), L^{p}\left(\mathbb{R}^{2}, \mathbb{R}^{2}\right)\right), \\
\Psi_{4}(\theta):= & \left(L_{\theta}\right)^{-1}\left(\frac{1}{2} I-K_{i \omega, \theta}^{*}\right)^{-1} L_{\theta}=\left[\left(L_{\theta}\right)^{-1}\left(\frac{1}{2} I-K_{i \omega, \theta}^{*}\right) L_{\theta}\right] \\
& \in \mathcal{L}\left(L^{p}\left(\mathbb{R}^{2}\right), L^{p}\left(\mathbb{R}^{2}\right)\right), \\
\Psi_{5}(\theta):= & \omega\left(L_{\theta}\right)^{-1}\left\langle\nu_{\theta}, \omega S_{i \omega, \theta} L_{\theta}\right\rangle \in \mathcal{L}\left(L^{p}\left(\mathbb{R}^{2}, \mathbb{R}^{2}\right), L^{p}\left(\mathbb{R}^{2}\right)\right), \\
\Psi_{6}(\theta):= & \left(L_{\theta}\right)^{-1}\left\langle\nu_{\theta}, \operatorname{curl} S_{i \omega, \theta} L_{\theta}\right\rangle \in \mathcal{L}\left(L^{p}\left(\mathbb{R}^{2}, \mathbb{R}^{2}\right), L^{p}\left(\mathbb{R}^{2}\right)\right) .
\end{aligned}
$$

Going further, these claims are going to be simple consequences of the fact that

$$
\left\|\frac{d}{d \theta} \Psi_{j}(\theta)\right\|_{\mathcal{L}\left(L^{p}, L^{p}\right)} \leq C<+\infty, \text { for } j=1,2, \ldots, 6 .
$$

This, in turn, can be verified based on the definitions of $L_{\theta}$ and $\varphi_{\theta}$, the identity (4.15), Proposition 2.1, (2.7), (2.12), the fact that taking the inverse is a continuous operation on the space of linear isomorphisms, and the detailed analysis of how the norm of a Calderón-Zygmund type operator defined on the graph of a Lipschitz function $\phi$ depends on $\|\nabla \phi\|_{L^{\infty}}$ (a good reference in this regard is [1]). As far as (4.26) is concerned, the interested reader is also referred to [16, 7] for more details in similar circumstances. 
Furthermore, an estimate from below - similar to (4.10) - holds for each $\Lambda_{\theta}$, $\theta \in[0,1]$. As a consequence, if $p=2$, then $\Lambda_{\theta}$ is semi-Fredholm for each $\theta \in[0,1]$ and

$$
\operatorname{index} \Lambda=\operatorname{index} \Lambda_{1}=\operatorname{index} \Lambda_{0} .
$$

Step III. We now claim that $\Lambda_{0}$ is onto whenever $1<p<2+\varepsilon$. To this end, suppose that

$$
\varphi\left(x_{1}, x_{2}\right)=\kappa x_{1} \text { on the set }\left\{\left(x_{1}, x_{2}\right): x_{1}>\psi\left(x_{2}\right)\right\} .
$$

Let $\nu_{h}:=(-\kappa, 0,1) / \sqrt{1+\kappa^{2}}$ be the unit normal to the plane $\Pi_{h}:=\left\{\left(x_{1}, x_{2}, \kappa x_{1}\right)\right.$ : $\left.x_{1}, x_{2} \in \mathbb{R}\right\}$ and denote by

$$
R: \mathbb{R}^{3} \longrightarrow \mathbb{R}^{3}, \quad R(x):=x-2\left\langle x, \nu_{h}\right\rangle \nu_{h},
$$

the reflection across $\Pi_{h}$. From (4.28) we see that $\Sigma^{h} \subseteq \Pi_{h}$ so that $R \equiv i d$ on $\Sigma^{h}$. The claim that we make at this stage is that if $f \in L_{t}^{p, \operatorname{Div}}\left(\Sigma^{e}\right)$, then the function

$$
F:=\left\{\begin{array}{l}
f \text { on } \Sigma^{e}, \\
-R f R \text { on } R \Sigma^{e},
\end{array}\right.
$$

satisfies

$$
F \in L_{t}^{p, \text { Div }}\left(\Sigma^{e} \sqcup R \Sigma^{e}\right) \text { and }\|F\|_{L_{t}^{p, \text { Div }}\left(\Sigma^{e} \sqcup R \Sigma^{e}\right)} \leq C\|f\|_{L_{t}^{p, \text { Div }}\left(\Sigma^{e}\right)} .
$$

Indeed, it is immediate that $F \in L_{t}^{p}\left(\Sigma^{e} \sqcup R \Sigma^{e}\right)$ and that $\|F\|_{L^{p}\left(\Sigma^{e} \sqcup R \Sigma^{e}, \mathbb{R}^{3}\right)} \leq$ $C\|f\|_{L^{p}\left(\Sigma^{e}, \mathbb{R}^{3}\right)}$. There remains to compute and estimate the surface-divergence of $F$. For this, we shall employ Gauss' identity to the effect that

$$
\int_{\Sigma}\left\langle f, \nabla_{\tan } \eta\right\rangle d \sigma=-\int_{\Sigma}(\operatorname{Div} f) \eta d \sigma+\oint_{\partial \Sigma}\langle f, \gamma\rangle \eta d s
$$

provided the following hold. First, $\Sigma$ is a (reasonably smooth) surface whose area element is denoted by $d \sigma$. Its boundary, $\partial \Sigma$, has $d s$ as its arc-length and $\gamma$ as its unit normal. Second, $f$ is a vector field tangent to $\Sigma$ and $\eta$ is a scalar-valued function defined on $\Sigma$. Both $f$ and $\eta$ aare assumed to be sufficiently well-behaved.

Pick an arbitrary, Lipschitz continuous, compactly supported, scalar-valued function $\eta$, defined on $\Sigma^{e} \sqcup R \Sigma^{e}$, and recall that $\Gamma:=\partial \Sigma^{e}=\partial \Sigma^{h}$. Then (temporarily ignoring smoothness issues - these can be dealt with via a limiting argument) we may write

$$
\begin{aligned}
& \int_{\Sigma^{e} \sqcup R \Sigma^{e}}\left\langle F, \nabla_{\tan } \eta\right\rangle d \sigma=\int_{\Sigma^{e}}\langle f, \nabla \eta\rangle d \sigma-\int_{R \Sigma^{e}}\langle R f R, \nabla \eta\rangle d \sigma \\
& =\int_{\Sigma^{e}}\langle f, \nabla \eta\rangle d \sigma-\int_{\Sigma^{e}}\langle f, \nabla(\eta \circ R)\rangle d \sigma \\
& =-\int_{\Sigma^{e}}(\operatorname{Div} f) \eta d \sigma+\oint_{\Gamma}\langle f, \gamma\rangle \eta d s+\int_{\Sigma^{e}}(\operatorname{Div} f)(\eta \circ R) d \sigma-\oint_{\Gamma}\langle f, \gamma\rangle(\eta \circ R) d s \\
& =-\int_{\Sigma^{e}}(\operatorname{Div} f) \eta d \sigma+\int_{\Sigma^{e}}(\operatorname{Div} f)(\eta \circ R) d \sigma
\end{aligned}
$$




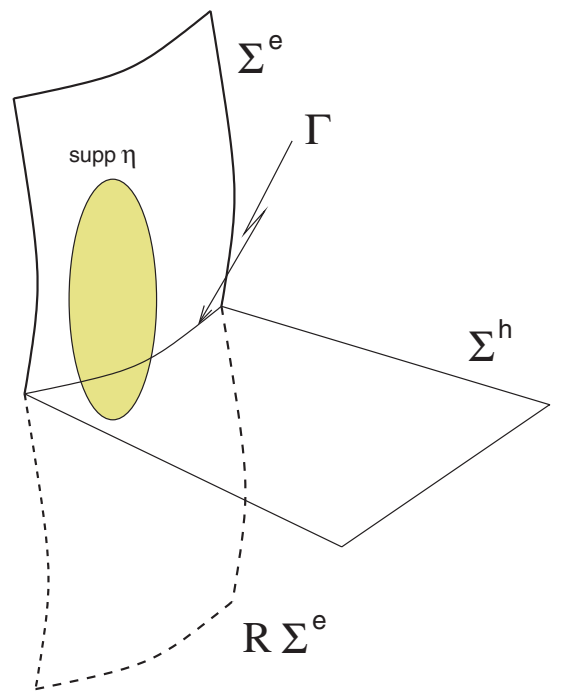

FIGURE 2

as the two path integrals cancel each other, due to the fact that $R \equiv i d$ on $\Gamma \subset \Pi_{h}$. Consequently,

$$
\operatorname{Div} F=\left\{\begin{array}{l}
\operatorname{Div} f \text { on } \Sigma^{e}, \\
-(\operatorname{Div} f) \circ R \text { on } R \Sigma^{e},
\end{array}\right.
$$

is in $L^{p}\left(\Sigma^{e} \sqcup R \Sigma^{e}\right)$ and satisfies $\|\operatorname{Div} F\|_{L^{p}\left(\Sigma^{e} \sqcup R \Sigma^{e}\right)} \leq 2\|\operatorname{Div} f\|_{L^{p}\left(\Sigma^{e}\right)}$, as desired.

To continue, we note that for general vector fields $f, g$,

$$
\operatorname{curl}(R f R)=-R(\operatorname{curl} f) R \text { and }(R f R) \times(R g R)=-R(f \times g) R .
$$

Furthermore, if we set $\Omega_{R}:=\Omega \cup \Sigma^{h} \cup R \Omega$, then $\Omega_{R}$ is a Lipschitz domain invariant under the reflection $R$ and $R \nu R=\nu$ on $\partial \Omega_{R}$.

Now fix $f \in L_{t}^{p \text {,Div }}\left(\Sigma^{e}\right)$ and define $F \in L_{t}^{p \text {,Div }}\left(\Sigma^{e} \sqcup R \Sigma^{e}\right)$ as in (4.30). Next, let $(E, H)$ solve

$$
\left\{\begin{array}{l}
\operatorname{curl} E=-\omega H \text { in } \Omega_{R}, \\
\operatorname{curl} H=\omega E \text { in } \Omega_{R}, \\
\nu \times\left. E\right|_{\partial \Omega_{R}}=F \text { on } \partial \Omega_{R}, \\
E^{*}, H^{*} \in L^{p}\left(\partial \Omega_{R}\right) .
\end{array}\right.
$$

If we now introduce $\hat{E}:=R E R$ and $\hat{H}:=-R H R$ in $\Omega_{R}$, then $(\hat{E}, \hat{H})$ solve a boundary problem similar to (4.36) except that, this time, the boundary data is $\nu \times(R E R)=(R \nu R) \times(R E R)=-R(\nu \times E) R=-R F R$. However, since $-R F R=F$, from the uniqueness part for (1.1) it follows that $\hat{E}=E$ and $\hat{H}=H$ in $\Omega_{R}$. In particular, on $\Sigma^{h}, H=-R H$, i.e., $H$ is normal to the support plane $\Pi_{h}$ and, ultimately, $\nu \times H=0$ on $\Sigma^{h}\left(\subseteq \Pi_{h}\right)$. Hence, $g:=\nu \times\left(\left.H\right|_{\partial \Omega}\right) \in L_{t, 0}^{p, \text { Div }}\left(\Sigma^{e}\right)$ 
and $\Lambda_{0}(g)=f$. Since $f \in L_{t}^{p \text {,Div }}\left(\Sigma^{e}\right)$ was arbitrary, this finishes the proof of the ontoness of the operator $\Lambda_{0}$.

Step IV. The final claim is that $\Lambda=\Lambda_{1}$ is invertible for $2-\varepsilon<p<2+\varepsilon$. Indeed, when $p=2$, the fact that $\Lambda_{0}$ is invertible follows from Steps I and III. Hence, thanks to (4.27), $\Lambda_{1}$ is Fredholm with index zero. Since it is also one-to-one, by (4.10), it follows that $\Lambda$ is an isomorphism for $p=2$. Finally, passing from $p=2$ to $|p-2|<\varepsilon$ can be done via functional analysis, by invoking certain well-known stability results.

\section{WELL-POSEDNESS FOR BOUNDED LIPSCHITZ DOMAINS}

In this section we prove Theorem 1.1 as stated. In a first stage, we assume that $k=i \omega$ for some large $\omega>0$ and seek to construct an "approximate" solution to (1.7) when $p=2$.

We begin by reviewing the assumptions made on the bounded Lipschitz domain $\Omega$. Assume that $\partial \Omega=\Sigma^{e} \sqcup \Sigma^{h}$ and that there exist a finite collection of points $\left\{x_{j}\right\}_{j}$ and $r>0$ with $\partial \Omega \subset \bigcup_{j} B_{r}\left(x_{j}\right)$, such that for each $j$ the following holds. There exists a domain $\Omega_{j}$ with boundary decomposition $\Sigma_{j}^{e}, \Sigma_{j}^{h}$, which are rigid motions of the graph domains satisfying (4.6)-(4.7), so that

$$
\begin{aligned}
& \Omega \cap B_{2 r}\left(x_{j}\right)=\Omega_{j} \cap B_{2 r}\left(x_{j}\right), \\
& \Sigma^{e} \cap B_{2 r}\left(x_{j}\right)=\Sigma_{j}^{e} \cap B_{2 r}\left(x_{j}\right), \\
& \Sigma^{h} \cap B_{2 r}\left(x_{j}\right)=\Sigma_{j}^{h} \cap B_{2 r}\left(x_{j}\right) .
\end{aligned}
$$

Next, let $\left\{\xi_{j}\right\}_{j}$ be a smooth partition of unity, subordinate to the cover $\left\{B_{r}\left(x_{j}\right)\right\}_{j}$ of $\partial \Omega$ and, for each $j$, pick $\eta_{j} \in C^{\infty}\left(B_{2 r}\left(x_{j}\right)\right)$ with $\eta_{j} \equiv 1$ on $\operatorname{supp} \xi_{j}$. Relying on the results from the previous section, for each $j$ solve

$$
\left\{\begin{array}{l}
\operatorname{curl} E_{j}+\omega H_{j}=0 \text { in } \Omega_{j}, \\
\operatorname{curl} H_{j}-\omega E_{j}=0 \text { in } \Omega_{j}, \\
\nu_{j} \times\left. E_{j}\right|_{\Sigma_{j}^{e}}=\xi_{j} f \text { on } \Sigma_{j}^{e}, \\
\nu_{j} \times\left. H_{j}\right|_{\Sigma_{j}^{h}}=\xi_{j} g \text { on } \Sigma_{j}^{h}, \\
E_{j}^{*}, H_{j}^{*} \in L^{2}\left(\partial \Omega_{j}\right),
\end{array}\right.
$$

where $\Omega_{j}, \Sigma_{j}^{e}, \Sigma_{j}^{h}$ are as in (5.1) and $\nu_{j}$ is the unit normal to $\partial \Omega_{j}$. In particular, our analysis from $\S 4$ gives

$$
\begin{aligned}
\int_{\partial \Omega_{j}}\left[\left|E_{j}^{*}\right|^{2}+\left|H_{j}^{*}\right|^{2}\right] d \sigma_{j} & \leq C\left(\int_{\Sigma^{e}}|f|^{2} d \sigma+\frac{1}{\omega^{2}} \int_{\Sigma^{e}}|\operatorname{Div} f|^{2} d \sigma\right)^{1 / 2} \\
& +C\left(\int_{\Sigma^{h}}|g|^{2} d \sigma+\frac{1}{\omega^{2}} \int_{\Sigma^{h}}|\operatorname{Div} g|^{2} d \sigma\right)^{1 / 2} .
\end{aligned}
$$

Set

$$
\hat{E}:=\sum_{j} \eta_{j} E_{j} \quad \text { and } \quad \hat{H}:=\sum_{j} \eta_{j} H_{j} \quad \text { in } \Omega
$$


so that

$$
\left.[\nu \times \hat{E}]\right|_{\Sigma^{e}}=f,\left.\quad[\nu \times \hat{H}]\right|_{\Sigma^{h}}=g
$$

and

$$
\left\{\begin{array}{l}
\operatorname{curl} \hat{E}+\omega \hat{H}=\sum_{j}\left(\nabla \eta_{j} \times E_{j}\right)=: K, \\
\operatorname{curl} \hat{H}-\omega \hat{E}=\sum_{j}\left(\nabla \eta_{j} \times H_{j}\right)=: J,
\end{array} \quad \text { in } \Omega .\right.
$$

Note that $\operatorname{div} K=\omega \sum_{j}\left\langle\nabla \eta_{j}, H_{j}\right\rangle$ and $\operatorname{div} J=-\omega \sum_{j}\left\langle\nabla \eta_{j}, E_{j}\right\rangle$ so that

$$
\begin{aligned}
\left\|J^{*}\right\|_{L^{2}(\partial \Omega)}+\left\|K^{*}\right\|_{L^{2}(\partial \Omega)} \leq & C\left(\int_{\Sigma^{e}}|f|^{2} d \sigma+\frac{1}{\omega^{2}} \int_{\Sigma^{e}}|\operatorname{Div} f|^{2} d \sigma\right)^{1 / 2} \\
& +C\left(\int_{\Sigma^{h}}|g|^{2} d \sigma+\frac{1}{\omega^{2}} \int_{\Sigma^{h}}|\operatorname{Div} g|^{2} d \sigma\right)^{1 / 2}
\end{aligned}
$$

and, by (3.12),

$$
\begin{aligned}
\|J\|_{L^{2}(\Omega)}+\omega^{-1} \| \operatorname{div} & J\left\|_{L^{2}(\Omega)}+\right\| K\left\|_{L^{2}(\Omega)}+\omega^{-1}\right\| \operatorname{div} K \|_{L^{2}(\Omega)} \\
\leq & C\left(\sum_{j}\left\|E_{j}\right\|_{L^{2}\left(\Omega_{j}\right)}+\sum_{j}\left\|H_{j}\right\|_{L^{2}\left(\Omega_{j}\right)}\right) \\
\leq & \frac{C}{\omega}\left(\int_{\Sigma^{e}}|f|^{2} d \sigma+\frac{1}{\omega^{2}} \int_{\Sigma^{e}}|\operatorname{Div} f|^{2} d \sigma\right)^{1 / 2} \\
& +\frac{C}{\omega}\left(\int_{\Sigma^{h}}|g|^{2} d \sigma+\frac{1}{\omega^{2}} \int_{\Sigma^{h}}|\operatorname{Div} g|^{2} d \sigma\right)^{1 / 2}
\end{aligned}
$$

Next, consider $\Pi_{\omega}:=\left(\Delta-\omega^{2}\right)^{-1}$, the Newtonian potential corresponding to the Helmholtz operator with wave number $k=i \omega, \omega>0$, and introduce

$$
\begin{aligned}
E^{\prime} & :=\left.\left[-\operatorname{curl} \Pi_{\omega} \tilde{J}-\omega^{-1} \nabla \operatorname{div} \Pi_{\omega} \tilde{K}+\omega \Pi_{\omega} \tilde{K}\right]\right|_{\Omega}, \\
H^{\prime} & :=\left.\left[-\operatorname{curl} \Pi_{\omega} \tilde{K}+\omega^{-1} \nabla \operatorname{div} \Pi_{\omega} \tilde{J}-\omega \Pi_{\omega} \tilde{J}\right]\right|_{\Omega},
\end{aligned}
$$

where the tilde denotes extension (to $\mathbb{R}^{3}$ ) by zero outside $\Omega$. Then, via a direct computation,

$$
\operatorname{curl} E^{\prime}+\omega H^{\prime}=K \text { and } \operatorname{curl} H^{\prime}-\omega E^{\prime}=J \text { in } \Omega .
$$

Furthermore, since

$$
\begin{aligned}
& \operatorname{div} \Pi_{\omega} \tilde{K}=\Pi_{\omega}(\widetilde{\operatorname{div} K})-\mathcal{S}_{i \omega}(\langle\nu, K\rangle) \quad \text { in } \Omega, \\
& \operatorname{div} \Pi_{\omega} \tilde{J}=\Pi_{\omega}(\widetilde{\operatorname{div} J})-\mathcal{S}_{i \omega}(\langle\nu, J\rangle) \quad \text { in } \Omega,
\end{aligned}
$$

we may write

$$
\begin{aligned}
& E^{\prime}=\left.\left[-\operatorname{curl} \Pi_{\omega} \tilde{J}-\omega^{-1} \nabla \Pi_{\omega}(\widetilde{\operatorname{div} K})+\omega^{-1} \nabla \mathcal{S}_{i \omega}(\langle\nu, K\rangle)+\omega \Pi_{\omega} \tilde{K}\right]\right|_{\Omega}, \\
& H^{\prime}=\left.\left[-\operatorname{curl} \Pi_{\omega} \tilde{K}+\omega^{-1} \nabla \Pi_{\omega}(\widetilde{\operatorname{div} J})-\omega^{-1} \nabla \mathcal{S}_{i \omega}(\langle\nu, K\rangle)-\omega \Pi_{\omega} \tilde{J}\right]\right|_{\Omega} .
\end{aligned}
$$


Thus, if we now set $E:=\hat{E}-E^{\prime}, H:=\hat{H}-H^{\prime}$, it follows that

$$
\begin{aligned}
& \operatorname{curl} E+\omega H=0, \quad \operatorname{curl} H-\omega E=0 \text { in } \Omega, \\
& \nu \times\left. E\right|_{\Sigma^{e}}-f=\nu \times\left. E^{\prime}\right|_{\Sigma^{e}},\left.\quad \operatorname{Div}(\nu \times E)\right|_{\Sigma^{e}}-\operatorname{Div} f=-\left.\left\langle\nu, \operatorname{curl} E^{\prime}\right\rangle\right|_{\Sigma^{e}}, \\
& \nu \times\left. H\right|_{\Sigma^{h}}-g=\nu \times\left. H^{\prime}\right|_{\Sigma^{h}},\left.\quad \operatorname{Div}(\nu \times H)\right|_{\Sigma^{h}}-\operatorname{Div} g=-\left.\left\langle\nu, \operatorname{curl} H^{\prime}\right\rangle\right|_{\Sigma^{h}} .
\end{aligned}
$$

Consequently, since for $\Omega$ bounded and $\omega \geq 1,1<p<\infty$,

$$
\begin{aligned}
\left\|\partial^{\alpha} \Pi_{\omega}\right\|_{\mathcal{L}\left(L^{p}\left(\mathbb{R}^{3}\right), L^{p}\left(\mathbb{R}^{3}\right)\right)} & \leq C \omega^{|\alpha|-2}, \quad \forall|\alpha| \leq 2 \\
\left\|\nabla S_{i \omega}\right\|_{\mathcal{L}\left(L^{p}(\partial \Omega), L^{p}(\partial \Omega)\right)} & \leq C \omega
\end{aligned}
$$

we may eventually conclude from (5.7), (5.15), (5.14), that for $\omega \geq 1$

$$
\begin{aligned}
\left\|E^{\prime}\right\|_{L^{2}\left(\partial \Omega, \mathbb{R}^{3}\right)} \leq & C\left\|\nabla \Pi_{\omega}\right\|_{\mathcal{L}\left(L^{2}\left(\mathbb{R}^{3}\right), L^{2}\left(\mathbb{R}^{3}\right)\right)}\left[\|J\|_{L^{2}(\Omega)}+\omega^{-1}\|\operatorname{div} K\|_{L^{2}(\Omega)}\right] \\
& +C \omega^{-1}\left\|\nabla S_{i \omega}\right\|_{\mathcal{L}\left(L^{2}(\partial \Omega), L^{2}(\partial \Omega)\right)}\|K\|_{L^{2}(\partial \Omega)} \\
& +C \omega\left\|\Pi_{\omega}\right\|_{\mathcal{L}\left(L^{2}\left(\mathbb{R}^{3}\right), L^{2}\left(\mathbb{R}^{3}\right)\right)}\|K\|_{L^{2}(\Omega)} \\
\leq & \frac{C}{\omega}\left\{\int_{\Sigma^{e}}|f|^{2} d \sigma+\frac{1}{\omega^{2}} \int_{\Sigma^{e}}|\operatorname{Div} f|^{2} d \sigma\right. \\
& \left.+\int_{\Sigma^{h}}|g|^{2} d \sigma+\frac{1}{\omega^{2}} \int_{\Sigma^{h}}|\operatorname{Div} g|^{2} d \sigma\right\}^{\frac{1}{2}} .
\end{aligned}
$$

Moreover, $\left\|H^{\prime}\right\|_{L^{2}(\partial \Omega)}$ can be estimated in a similar fashion. Next, curl $E^{\prime}=$ $-\omega H^{\prime}+K$ and curl $H^{\prime}=\omega E^{\prime}+J$ so that

$\left\|\operatorname{curl} E^{\prime}\right\|_{L^{2}\left(\partial \Omega, \mathbb{R}^{3}\right)}+\left\|\operatorname{curl} H^{\prime}\right\|_{L^{2}\left(\partial \Omega, \mathbb{R}^{3}\right)}$

$$
\leq C\left(\|f\|_{L^{2}\left(\Sigma^{e}, \mathbb{R}^{3}\right)}+\omega^{-1}\|\operatorname{Div} f\|_{L^{2}\left(\Sigma^{e}\right)}+\|g\|_{L^{2}\left(\Sigma^{h}, \mathbb{R}^{3}\right)}+\omega^{-1}\|\operatorname{Div} g\|_{L^{2}\left(\Sigma^{h}\right)}\right) .
$$

All in all,

$$
\begin{aligned}
\left\|\nu \times E^{\prime}\right\|_{L^{2}\left(\partial \Omega, \mathbb{R}^{3}\right)}+\omega^{-1}\left\|\operatorname{Div}\left(\nu \times E^{\prime}\right)\right\|_{L^{2}(\partial \Omega)} & \\
& +\left\|\nu \times H^{\prime}\right\|_{L^{2}\left(\partial \Omega, \mathbb{R}^{3}\right)}+\omega^{-1}\left\|\operatorname{Div}\left(\nu \times H^{\prime}\right)\right\|_{L^{2}(\partial \Omega)} \\
\leq & C \omega^{-1}\left(\|f\|_{L^{2}\left(\Sigma^{e}, \mathbb{R}^{3}\right)}+\omega^{-1}\|\operatorname{Div} f\|_{L^{2}\left(\Sigma^{e}\right)}+\|g\|_{L^{2}\left(\Sigma^{h}, \mathbb{R}^{3}\right)}+\omega^{-1}\|\operatorname{Div} g\|_{L^{2}\left(\Sigma^{h}\right)}\right),
\end{aligned}
$$

uniformly for $\omega$ large. Hence, finally,

$$
\begin{aligned}
& \int_{\Sigma^{e}}|\nu \times E-f|^{2} d \sigma+\frac{1}{\omega^{2}} \int_{\Sigma^{e}}|\operatorname{Div}(\nu \times E-f)|^{2} d \sigma \\
& +\int_{\Sigma^{h}}|\nu \times H-g|^{2} d \sigma+\frac{1}{\omega^{2}} \int_{\Sigma^{h}}|\operatorname{Div}(\nu \times H-g)|^{2} d \sigma \\
& \quad \leq \frac{C}{\omega^{2}}\left\{\int_{\Sigma^{e}}|f|^{2} d \sigma+\frac{1}{\omega^{2}} \int_{\Sigma^{e}}|\operatorname{Div} f|^{2} d \sigma+\int_{\Sigma^{h}}|g|^{2} d \sigma+\frac{1}{\omega^{2}} \int_{\Sigma^{h}}|\operatorname{Div} g|^{2} d \sigma\right\},
\end{aligned}
$$


uniformly for $\omega$ large. In turn, this implies that the "mixed tangential trace" operator

$$
\begin{aligned}
& \mathcal{T}: L_{t}^{2, \text { Div }}\left(\Sigma^{e}\right) \oplus L_{t}^{2, \text { Div }}\left(\Sigma^{h}\right) \longrightarrow L_{t}^{2, \text { Div }}\left(\Sigma^{e}\right) \oplus L_{t}^{2, \text { Div }}\left(\Sigma^{h}\right), \\
& \mathcal{T}(f, g):=\left(\nu \times\left. E\right|_{\Sigma^{e}}, \nu \times\left. H\right|_{\Sigma^{h}}\right),
\end{aligned}
$$

is invertible for $\omega>0$ large, since it satisfies

$$
\|\mathcal{T}-I\|_{\mathcal{L}\left(L_{t}^{2, \text { Div }}\left(\Sigma^{e}\right) \oplus L_{t}^{2, \text { Div }}\left(\Sigma^{h}\right), L_{t}^{2, \text { Div }}\left(\Sigma^{e}\right) \oplus L_{t}^{2, \text { Div }}\left(\Sigma^{h}\right)\right)} \leq C \omega^{-1}<1 .
$$

This shows that the mixed boundary value problem (1.7) is always well-posed if $p=2$ and $k=i \omega$ with $\omega>0$ large. Since, from [20, [18] we know that any two vector fields $E, H$ satisfying

$$
\left\{\begin{array}{l}
\operatorname{curl} E-i k H=0 \text { in } \Omega, \\
\operatorname{curl} H+i k E=0 \text { in } \Omega, \\
E^{*}, H^{*} \in L^{p}(\partial \Omega),
\end{array}\right.
$$

with $1<p<2+\varepsilon$, are always representable in the form

$$
\begin{aligned}
& E=\operatorname{curl} \mathcal{S}_{k} f, \text { in } \Omega, \\
& H=k^{2} \nu \times \mathcal{S}_{k} f+\nu \times \nabla \mathcal{S}_{k}(\operatorname{Div} f) \text { in } \Omega,
\end{aligned}
$$

for some (unique) $f \in L_{t}^{p \text {,Div }}(\partial \Omega)$, it follows that the singular integral operator

$$
\begin{aligned}
& T_{k}: L_{t}^{2, \text { Div }}(\partial \Omega) \longrightarrow L_{t}^{2, \text { Div }}\left(\Sigma^{e}\right) \oplus L_{t}^{2, \text { Div }}\left(\Sigma^{h}\right), \\
& T_{k} f:=\left(\left.\left[\frac{1}{2} I+M_{k}\right] f\right|_{\Sigma^{e}},\left.\left[k^{2} \nu \times S_{k} f+\nu \times \nabla S_{k}(\operatorname{Div} f)\right]\right|_{\Sigma^{h}}\right)
\end{aligned}
$$

is invertible if $k=i \omega$ with $\omega>0$ large enough.

On the other hand, it is possible to find a smooth vector field $\Theta$ such that (3.3) holds in this context (by patching together constant fields, suitably chosen for each graph domain via a partition of unity). Hence, the analogue of the Rellich estimate (3.11) is, in the present context,

$$
\begin{aligned}
\int_{\partial \Omega}\left\{|E|^{2}+|H|^{2}\right\} d \sigma \leq & C(\Omega, k) \int_{\Sigma^{e}}\left\{|\nu \times E|^{2}+|\operatorname{Div}(\nu \times E)|^{2}\right\} d \sigma \\
& +C(\Omega, k) \int_{\Sigma^{h}}\left\{|\nu \times H|^{2}+|\operatorname{Div}(\nu \times H)|^{2}\right\} d \sigma . \\
& +C(\Omega, k) \int_{\Omega}\left\{|E|^{2}+|H|^{2}\right\} d x .
\end{aligned}
$$

When written for $E, H$ of the form (5.22), this yields

$$
\begin{aligned}
\|f\|_{L_{t}^{2, \text { Div }}(\partial \Omega)} & \leq C\left\|\left(\frac{1}{2} I+M_{k}\right) f\right\|_{L_{t}^{2, \text { Div }}(\partial \Omega)}=C\|\nu \times E\|_{L_{t}^{2, \text { Div }}(\partial \Omega)} \\
& \leq C\|E\|_{L^{2}\left(\partial \Omega, \mathbb{R}^{3}\right)}+C\|H\|_{L^{2}\left(\partial \Omega, \mathbb{R}^{3}\right)} \\
& \leq C\left\|T_{k} f\right\|_{L_{t}^{2, \text { Div }}\left(\Sigma^{e}\right) \oplus L_{t}^{2, \text { Div }}\left(\Sigma^{h}\right)}+\|\operatorname{Comp} f\|,
\end{aligned}
$$

where Comp stands for a generic compact operator (here, contributed by the solid integrals in (5.24)). The first inequality above is nontrivial and has been established in 20]. In particular, the operator (5.23) is semi-Fredholm for any $\operatorname{Im} k \geq 0$. 
From the standard energy identity we also get that, if $\operatorname{Im} k>0$, then

$$
\int_{\Omega}\left\{|E|^{2}+|H|^{2}\right\} d x \leq C(\Omega, k)\left|\int_{\partial \Omega}\langle\nu \times E, H\rangle d \sigma\right|
$$

from which we may conclude that $T_{k}$ is one-to-one provided $\operatorname{Im} k>0$. Finally, the application

$$
\mathbb{C} \ni k \mapsto T_{k} \in \mathcal{L}\left(L_{t}^{p, \text { Div }}(\partial \Omega), L_{t}^{p, \text { Div }}\left(\Sigma^{e}\right) \oplus L_{t}^{p, \text { Div }}\left(\Sigma^{h}\right)\right)
$$

is continuous. In fact, it is not difficult to check that for any bounded Lipschitz domain $\Omega$ and any $1<p<\infty$,

$$
\left\|\frac{d}{d k} T_{k}\right\|_{\mathcal{L}\left(L^{p}, L^{p}\right)} \leq C(\Omega, p)<+\infty \text {, uniformly for } k \text { in compacta, }
$$

from which the claim made about (5.27) follows immediately.

To sum up, the invariance of index under continuous deformations within the class of semi-Fredholm operators yields that $T_{k}$ in (5.23) is Fredholm with index zero if $\operatorname{Im} k \geq 0$ and, further, invertible if $\operatorname{Im} k>0$. The extension to $p$ near 2 is then a consequence of this and known stability results ( since $L_{t}^{p \text {,Div }}(\partial \Omega), L_{t}^{p \text {,Div }}\left(\Sigma^{e}\right)$, $L_{t}^{p \text {,Div }}\left(\Sigma^{h}\right)$ are all complex interpolation scales; cf. 17. for more general results of this type).

\section{The HIGHER-DiMENSIONAL SETTING}

Here we present the proof of Theorem 1.2 Since this largely parallels the proof of Theorem 1.1 we sketch the main steps and only emphasize those aspects which require new tools and/or ideas.

For starters, the spaces involved in the formulation of (1.9) can be introduced in a thoroughly analogous fashion to (4.1). Next, given a bounded Lipschitz domain $\Omega$ in $\mathbb{R}^{n}, n \geq 2, k \in \mathbb{C}, \operatorname{Im} k \geq 0$ and $\ell \in\{0,1,2, \ldots, n\}$, it has been proved in [12] that one can find a $(\ell+1)$-form $E$ and a $\ell$-form $H$, smooth in $\Omega$ such that

$$
\left\{\begin{array}{l}
\delta E-i k H=0 \text { in } \Omega \\
d H+i k E=0 \text { in } \Omega \\
\left.\nu \wedge H\right|_{\partial \Omega}=g \in L_{n}^{p, d}\left(\partial \Omega, \Lambda^{\ell+1}\right), \\
E^{*}, H^{*} \in L^{p}(\partial \Omega)
\end{array}\right.
$$

obeying natural estimates. Therefore, much as in the three-dimensional setting, we may assume $g=0$ in (1.9), whenever convenient.

The relevant Rellich type identity, already proved in [12, amounts to the following. If $\left\{e_{j}\right\}_{j=1}^{n}$ is the standard orthonormal basis in $\mathbb{R}^{n}$, then, for any form $E \in C^{\infty}\left(\bar{\Omega}, \Lambda^{\ell}\right)$ and any vector field $\Theta=\left\{\Theta_{j}\right\}_{j}$ in $\mathbb{R}^{n}$, with smooth, real-valued components, we have

$$
\begin{gathered}
\operatorname{Re} \int_{\Omega} \frac{1}{2}|E|^{2} \operatorname{div} \Theta+\langle\delta E, \Theta \vee \bar{E}\rangle+\langle d E, \Theta \wedge \bar{E}\rangle-\sum_{j=1}^{n}\left\langle\nabla \Theta_{j} \vee E, e_{j} \vee \bar{E}\right\rangle \\
=\int_{\partial \Omega} \frac{1}{2}\left\{|\nu \wedge E|^{2}-|\nu \vee E|^{2}\right\}\langle\Theta, \nu\rangle d \sigma
\end{gathered}
$$


For a creased domain $\Omega$, this eventually leads to an estimate of the type

$$
\begin{aligned}
\int_{\partial \Omega}\left\{|E|^{2}+|H|^{2}\right\} d \sigma \leq & C \int_{\Sigma^{e}}\left\{|\nu \vee E|^{2}+\omega^{-2}\left|\delta_{\partial}(\nu \vee E)\right|^{2}\right\} d \sigma \\
& +C \int_{\Sigma^{h}}\left\{|\nu \wedge H|^{2}+\omega^{-2}\left|d_{\partial}(\nu \wedge H)\right|^{2}\right\} d \sigma,
\end{aligned}
$$

by reasoning much as in $\S 4$.

Once again, it is convenient to first consider the case when $\Omega$ is a graph domain and seek to construct a suitable lifting operator $L$ enjoying similar properties to those described in Proposition 2.1. In the context of differential forms of general degree, this is a more involved step, which requires a preliminary discussion.

Denote by Meas $\left(\partial \Omega, \Lambda^{\ell}\right)$ the vector space of $\Lambda^{\ell}$-valued functions defined on $\partial \Omega$. Similarly, Meas $\left(\partial \Omega, \Lambda^{\ell} T \partial \Omega\right)$ stands for the space of global, measurable sections in the vector bundle of $\ell$-forms on the Lipschitz manifold $\partial \Omega$. For each $x \in \partial \Omega$, we let $\mathrm{pr}_{x}$ stand for the orthogonal projection of $\mathbb{R}^{n}$ onto $T_{x} \partial \Omega$ and introduce

$$
\begin{aligned}
& \operatorname{pr}_{\star}: \text { Meas }\left(\partial \Omega, \Lambda^{\ell} T \partial \Omega\right) \longrightarrow \text { Meas }\left(\partial \Omega, \Lambda^{\ell}\right), \\
& \left(\operatorname{pr}_{\star} \omega\right)(x)\left(X_{1}, \ldots, X_{\ell}\right):=\omega(x)\left(\operatorname{pr}_{x} X_{1}, \ldots, \operatorname{pr}_{x} X_{\ell}\right)
\end{aligned}
$$

for each $\omega \in$ Meas $\left(\partial \Omega, \Lambda^{\ell} T \partial \Omega\right)$, a.e. $x \in \partial \Omega$ and each $X_{1}, \ldots, X_{\ell} \in \mathbb{R}^{n}$. We shall also work with

$$
j_{\star}: \operatorname{Meas}\left(\partial \Omega, \Lambda^{\ell}\right) \longrightarrow \operatorname{Meas}\left(\partial \Omega, \Lambda^{\ell} T \partial \Omega\right)
$$

defined at a.e. $x \in \partial \Omega$ by

$$
\left(j_{\star} w\right)(x)\left(X_{1}, \ldots, X_{\ell}\right):=w(x)\left(\iota\left(X_{1}\right), . ., \iota\left(X_{\ell}\right)\right),
$$

where $w \in \operatorname{Meas}\left(\partial \Omega, \Lambda^{\ell}\right), X_{1}, \ldots, X_{\ell} \in T_{x} \partial \Omega$, and $\iota$ is the inclusion of $T_{x} \partial \Omega$ into $\mathbb{R}^{n}$. The next two lemmas have been established in [19].

Lemma 6.1. If $\omega \in \operatorname{Meas}\left(\partial \Omega, \Lambda^{\ell} T \partial \Omega\right)$, then $\operatorname{pr}_{\star} \omega$ is tangential and $j_{\star} \mathrm{pr}_{\star} \omega=\omega$. Conversely, if $\omega \in \operatorname{Meas}\left(\partial \Omega, \Lambda^{\ell}\right)$, then $\operatorname{pr}_{\star} j_{\star} \omega=\omega_{t}$, the tangential component of $\omega$. In fact, for each $1<p<\infty$ and $0 \leq \ell \leq n$, the mappings

$$
\begin{aligned}
& j_{\star}: L_{t}^{p}\left(\partial \Omega, \Lambda^{\ell}\right) \longrightarrow L^{p}\left(\partial \Omega, \Lambda^{\ell} T \partial \Omega\right), \\
& \operatorname{pr}_{\star}: L^{p}\left(\partial \Omega, \Lambda^{\ell} T \partial \Omega\right) \longrightarrow L_{t}^{p}\left(\partial \Omega, \Lambda^{\ell}\right)
\end{aligned}
$$

are isometric inverses of each other.

Let $d_{\partial \Omega}$ denote the intrinsic exterior derivative operator on the Lipschitz manifold $\partial \Omega$. For each $1<p<\infty$ and $0 \leq \ell \leq n$ we consider the maximal closed (unbounded) operator defined by $d_{\partial \Omega}$ on $L^{p}\left(\partial \Omega, \Lambda^{\ell} T \partial \Omega\right)$. Its domain is

$$
\operatorname{Dom}_{\ell, p}\left(d_{\partial \Omega}\right):=\left\{\omega \in L^{p}\left(\partial \Omega, \Lambda^{\ell} T \partial \Omega\right): d_{\partial \Omega} \omega \in L^{p}\left(\partial \Omega, \Lambda^{l+1} T \partial \Omega\right)\right\} .
$$

Lemma 6.2. For $f \in L_{n}^{p, d}\left(\partial \Omega, \Lambda^{\ell}\right)$ we have $j_{\star}(\nu \vee f) \in \operatorname{Dom}_{\ell-1, p}\left(d_{\partial \Omega}\right)$ and

$$
d_{\partial \Omega}\left(j_{\star}(\nu \vee f)\right)=-j_{\star}\left(\nu \vee d_{\partial} f\right) .
$$

Conversely, if $f \in \operatorname{Dom}_{\ell, p}\left(d_{\partial \Omega}\right)$, then $\nu \wedge \operatorname{pr}_{\star} f \in L_{n}^{p, d}\left(\partial \Omega, \Lambda^{\ell+1}\right)$ and

$$
d_{\partial}\left(\nu \wedge \operatorname{pr}_{\star} f\right)=-\nu \wedge \operatorname{pr}_{\star} d_{\partial \Omega} f .
$$

In particular, we have that

$$
d_{\partial}=-\nu \wedge \operatorname{pr}_{\star} d_{\partial \Omega} j_{\star} \nu \vee \cdot \quad \text { and } \quad d_{\partial \Omega}=-j_{\star} \nu \vee d_{\partial}\left(\nu \wedge \mathrm{pr}_{\star}\right)
$$

in the sense of the composition of unbounded operators. 
Now consider the case when $\partial \Omega$ is the graph of the Lipschitz function $\varphi: \mathbb{R}^{n} \rightarrow$ $\mathbb{R}$. Let $\pi: \mathbb{R}^{n} \rightarrow \mathbb{R}^{n-1}$ be the projection onto the first $(n-1)$-coordinates, i.e., $\pi\left(x^{\prime}, x_{n}\right)=x^{\prime}$. When restricted to $\partial \Omega$, this becomes a bi-Lipschitz homeomorphism with inverse $\pi^{-1}\left(x^{\prime}\right)=\left(x^{\prime}, \varphi\left(x^{\prime}\right)\right), x^{\prime} \in \mathbb{R}^{n-1}$. We denote by $\pi^{*}$ : Meas $\left(\mathbb{R}^{n-1}, \Lambda^{\ell}\right) \rightarrow$ Meas $\left(\partial \Omega, \Lambda^{\ell} T \partial \Omega\right)$ and $\left(\pi^{-1}\right)^{*}:$ Meas $\left(\partial \Omega, \Lambda^{\ell} T \partial \Omega\right) \rightarrow$ Meas $\left(\mathbb{R}^{n-1}, \Lambda^{\ell}\right)$ the corresponding pull-back maps.

Proposition 6.3. For each $0 \leq \ell \leq n$, the lifting map

$$
L_{\ell}: \operatorname{Meas}\left(\mathbb{R}^{n-1}, \Lambda^{\ell} \mathbb{R}^{n-1}\right) \longrightarrow \operatorname{Meas}\left(\partial \Omega, \Lambda^{n-\ell-1}\right), \quad L_{\ell} f:=*\left[\nu \wedge \operatorname{pr}_{\star} \pi^{*}(f)\right]
$$

induces two linear isomorphisms:

$$
L_{\ell}: L^{p}\left(\mathbb{R}^{n-1}, \Lambda^{\ell} \mathbb{R}^{n-1}\right) \longrightarrow L_{t}^{p}\left(\partial \Omega, \Lambda^{n-\ell-1}\right),
$$

as well as

$$
\begin{aligned}
L_{\ell}:\{f & \left.\in L^{p}\left(\mathbb{R}^{n-1}, \Lambda^{\ell} \mathbb{R}^{n-1}\right): d_{\mathbb{R}^{n-1}} f \in L^{p}\left(\mathbb{R}^{n-1}, \Lambda^{\ell+1} \mathbb{R}^{n-1}\right)\right\} \\
& \longrightarrow L_{t}^{p, \delta}\left(\partial \Omega, \Lambda^{n-\ell-1}\right),
\end{aligned}
$$

where $d_{\mathbb{R}^{n-1}}$ denotes the exterior derivative operator in $\mathbb{R}^{n-1}$. In each case, its inverse is given by

$$
L_{\ell}^{-1} f=(-1)^{\ell(n-\ell-1)}\left(\pi^{-1}\right)^{*}\left[j_{\star}(\nu \vee * f)\right] .
$$

Furthermore, for each $f \in L^{p}\left(\mathbb{R}^{n-1}, \Lambda^{\ell} \mathbb{R}^{n-1}\right)$ with $d_{\mathbb{R}^{n-1}} f \in L^{p}\left(\mathbb{R}^{n-1}, \Lambda^{\ell+1} \mathbb{R}^{n-1}\right)$,

$$
\delta_{\partial} L_{\ell} f=(-1)^{n-\ell-1} L_{\ell+1}\left(d_{\mathbb{R}^{n-1}} f\right) .
$$

Proof. To begin with, $\left|L_{\ell} f\right|=\left|*\left[\nu \wedge \operatorname{pr}_{\star} \pi^{*}(f)\right]\right|=\left|\nu \wedge \operatorname{pr}_{\star} \pi^{*}(f)\right|=\left|\pi^{*}(f)\right| \approx|f|$, thanks to Lemma 6.1 and the fact that the Hodge star-operator is an isometry. This ensures that the operator in (6.13) is well-defined and bounded.

Next, since $\delta_{\partial}(* g)=(-1)^{\ell+1} *\left(d_{\partial} g\right)$ for every $g \in L_{n}^{p, d}\left(\partial \Omega, \Lambda^{\ell}\right)$, it follows that (6.17)

$$
\begin{aligned}
\delta_{\partial} L_{\ell} f & =\delta_{\partial}\left[*\left[\nu \wedge \mathrm{pr}_{\star} \pi^{*}(f)\right]\right]=(-1)^{n-\ell} * d_{\partial}\left[\nu \wedge \mathrm{pr}_{\star} \pi^{*}(f)\right] \\
& =(-1)^{n-\ell-1} * \nu \wedge \mathrm{pr}_{\star} d_{\partial \Omega} j_{\star} \nu \vee\left[\nu \wedge \mathrm{pr}_{\star} \pi^{*}(f)\right] \\
& =(-1)^{n-\ell-1} * \nu \wedge \operatorname{pr}_{\star} d_{\partial \Omega} j_{\star} \pi^{*}(f)=(-1)^{n-\ell-1} * \nu \wedge \mathrm{pr}_{\star} d_{\partial \Omega} \pi^{*}(f) \\
& =(-1)^{n-\ell-1} * \nu \wedge \operatorname{pr}_{\star} \pi^{*}\left(d_{\mathbb{R}^{n-1}} f\right)=(-1)^{n-\ell-1} L_{\ell+1}\left(d_{\mathbb{R}^{n-1}} f\right),
\end{aligned}
$$

based on Lemma 6.1, Lemma 6.2, and the fact that the exterior derivative operator commutes with pull-back. This justifies (6.16).

Finally, that (6.15) is the inverse of (6.12) follows once again from Lemma 6.1 via straightforward calculations.

With Proposition 6.3 in hand, the same type of deformation argument as in Step II of $\S 4$ can be performed, given that the integral operators involved -cf. (2.35), (2.36), (2.49), (2.50) - have the same nature as those encountered in the three-dimensional setting.

Next, we explain how the reflection argument from (4.28)-(4.36) should be carried out in the present context. Much as in $\S 4$, we flatten $\Sigma^{h}$ and let $R$ stand for the reflection operator across the supporting hyperplane $\Pi_{h}:=\left\{\left(x_{1}, x^{\prime \prime}, \kappa x_{1}\right): x_{1} \in\right.$ $\left.\mathbb{R}, x^{\prime \prime} \in \mathbb{R}^{n-2}\right\}$. Denote by $R^{*}$ the associated pull-back map and let $\Omega_{R}$ retain the 
same significance as in $\S 4$. Then $R \equiv i d$ on $\Sigma^{h}$ and $R^{*} \nu=\nu$ on $\partial \Omega_{R}$. Other useful properties are as follows:

$$
\begin{aligned}
& *\left(R^{*} E\right)=-R^{*}(* E), \quad\left\langle R^{*} E, R^{*} F\right\rangle=\langle E, F\rangle \circ R, \\
& R^{*}(\alpha \wedge E)=\left(R^{*} \alpha\right) \wedge(R * E), \quad R^{*}(\alpha \vee E)=\left(R^{*} \alpha\right) \vee(R * E), \\
& d R^{*} E=R^{*} d E, \quad \delta R^{*} E=R^{*} \delta E, \quad R^{*} R^{*}=i d,
\end{aligned}
$$

for any differential forms $E, F$ and any vector field $\alpha$. In particular, if $(E, H)$ solve the Maxwell system with boundary datum $\nu \wedge H=F$ on $\Omega_{R}$, then $\left(R^{*} E, R^{*} H\right)$ also solve the Maxwell system with boundary datum $\nu \wedge R^{*} H=R^{*} F$ on $\Omega_{R}$. Thus, if $R^{*} F=-F$, then necessarily $E=-R^{*} E$ and $H=-R^{*} H$ in $\Omega_{R}$, granted the uniqueness results proved in [12. Going further, the latter readily implies $\nu \wedge H=0$ on $\Sigma^{h}\left(\subset \Pi_{h}\right)$, as desired. In our case,

$$
F:=\left\{\begin{array}{l}
f \text { on } \Sigma^{e} \\
-R^{*} f \text { on } R \Sigma^{e}
\end{array}\right.
$$

which is the analogue of (4.30). From this, it is immediate that $R^{*} F=-F$. There remains to check that

$$
\begin{aligned}
& f \in L_{t}^{p, \delta}\left(\Sigma^{h}, \Lambda^{\ell}\right) \Longrightarrow F \in L_{t}^{p, \delta}\left(\Sigma^{h} \sqcup R \Sigma^{h}, \Lambda^{\ell}\right) \\
& \text { and }\|F\|_{L_{t}^{p, \delta}\left(\Sigma^{h} \sqcup R \Sigma^{h}, \Lambda^{\ell}\right)} \leq C\|f\|_{L_{t}^{p, \delta}\left(\Sigma^{h}, \Lambda^{\ell}\right)} .
\end{aligned}
$$

This step can be executed much as we did in $\S 4$, granted that a suitable generalization of Gauss' formula (4.32) is available in this context. Nonetheless, this has already been taken care of in 21], from which we quote the following result.

Proposition 6.4. Let $\Sigma$ be a surface of co-dimension one in $\mathbb{R}^{n}$ and denote by $\gamma$ and $d s$, respectively, the unit normal and the area element on $\partial \Sigma$. Also, let $\iota: \partial \Sigma \hookrightarrow \mathbb{R}^{n}$ be the natural inclusion. Then for every $f: \bar{\Sigma} \rightarrow \Lambda^{\ell}$ which is tangential to $\Sigma$ and every $g: \bar{\Sigma} \rightarrow \Lambda^{\ell-1}$ there holds

$$
\int_{\Sigma}\langle f, d g\rangle d \sigma-\int_{\Sigma}\left\langle\delta_{\partial} f, g\right\rangle d \sigma=\oint_{\partial \Sigma}\langle f, \gamma \wedge g\rangle d s=\int_{\partial \Sigma} \iota^{*}(g \wedge *(\nu \wedge f)) .
$$

Granted this integral identity, (6.20) follows essentially as before. The remaining steps in the proof of Theorem 1.2 closely parallel those taken in $\S 4-\S 5$, granted that representation formulas in terms of singular integral operators are available; cf. the operator theory reviewed in $\S 2.2$.

\section{REFERENCES}

[1] A. Alonso Rodríguez and M. Raffetto, Unique solvability for electromagnetic boundary value problems in the presence of partly lossy inhomogeneous anisotropic media and mixed boundary conditions, Math. Models Methods Appl. Sci., 13 (2003), no. 4, 597-611. MR1976304 (2004c:35396)

[2] G.A.Baker and J. Dodziuk, Stability of spectra of Hodge-de Rham Laplacians, Math. Z., 224 (1997), no. 3, 327-345. MR1439194 (98h:58191)

[3] R. Brown, The mixed problem for Laplace's equation in a class of Lipschitz domains, Comm. Partial Differential Equations, 19 (1994), no. 7-8, 1217-1233. MR.1284808 (95i:35059)

[4] F. Cakoni and D. Colton, Mixed boundary value problems in inverse electromagnetic scattering, Proceedings of the 6th International Workshop on Mathematical Methods in Scattering Theory and Biomedical Engineering, Tsepelovo, 2003. 
[5] F. Cakoni, D. Colton and P. Monk, Electromagnetic inverse scattering problem for partially coated Lipschitz Domains, to appear in Proc. Royal Society of Edinburgh. MR2079799 (2005g:78016)

[6] R.R. Coifman, A. McIntosh and Y. Meyer, L'intégrale de Cauchy définit un opérateur borné sur $L^{2}$ pour les courbes lipschitziennes, Ann. of Math. (2), 116 (1982), no. 2, 361-387. MR672839(84m:42027)

[7] B. Dahlberg and C. Kenig, Hardy spaces and the $L^{p}-$ Neumann problem for Laplace's equation in a Lipschitz domain, Ann. of Math., 125 (1987), 437-465. MR890159 (88d:35044)

[8] E.B. Fabes, M. Jodeit, Jr. and N.M. Rivière, Potential techniques for boundary value problems on $C^{1}$-domains, Acta Math., 141 (1978), no. 3-4, 165-186. MR501367(80b:31006)

[9] P. Fernandes and G. Gilardi, Magnetostatic and electrostatic problems in inhomogeneous anisotropic media with irregular boundary and mixed boundary conditions, Math. Models Methods Appl. Sci., 7 (1997), no. 7, 957-991. MR.1479578 (98h:78006)

[10] H.G.Garnir, Les problémes aux limites de la physique mathématique, Basel, Birkhäuser Verlag, 1958. MR0104054 (21:2816)

[11] S. Hofmann, On singular integrals of Calderón-type in $\mathbb{R}^{n}$, and BMO, Rev. Mat. Iberoamericana, 10 (1994), no. 3, 467-505. MR.1308701 (96d:42019)

[12] B. Jawerth and M. Mitrea, Higher-dimensional electromagnetic scattering theory on $C^{1}$ and Lipschitz domains, Amer. J. Math., 117 (1995), no. 4, 929-963. MR1342836 (96h:35143)

[13] F. Jochmann, Existence of weak solutions of the drift diffusion model coupled with Maxwell's equations, J. Math. Anal. Appl., 204 (1996), no. 3, 655-676. MR.1422765 (98e:35030)

[14] F. Jochmann, Uniqueness and regularity for the two-dimensional drift-diffusion model for semiconductors coupled with Maxwell's equations, J. Differential Equations, 147 (1998), no. 2, 242-270. MR 1634016 (99g:35125)

[15] F. Jochmann, Regularity of weak solutions of Maxwell's equations with mixed boundaryconditions, Math. Methods Appl. Sci., 22 (1999), no. 14, 1255-1274. MR.1710708 (2000g:78008)

[16] C.E. Kenig, Recent progress on boundary value problems on Lipschitz domains, Pseudodifferential operators and applications (Notre Dame, Ind., 1984), pp. 175-205, Proc. Sympos. Pure Math., 43, Amer. Math. Soc., Providence, RI, 1985. MR812291(87e:35029)

[17] D. Mitrea and M. Mitrea, Finite energy solutions of Maxwell's equations and constructive Hodge decompositions on nonsmooth Riemannian manifolds, J. Funct. Anal., 190 (2002), no. 2, 339-417. MR 1899489 (2003m:58002)

[18] D. Mitrea, M. Mitrea and J. Pipher, Vector potential theory on nonsmooth domains in $\mathbb{R}^{3}$ and applications to electromagnetic scattering, J. Fourier Anal. Appl., 3 (1997), no. 2, 131192. MR.1438894 (99e:31009)

[19] D. Mitrea, M. Mitrea and M. Taylor, Layer Potentials, the Hodge Laplacian, and Global Boundary Problems in Nonsmooth Riemannian Manifolds, Mem. Amer. Math. Soc., Vol. 150 No. 713, 2001. MR1809655 (2002g:58026)

[20] M. Mitrea, The method of layer potentials in electromagnetic scattering theory on nonsmooth domains, Duke Math. J., 77 (1995), no. 1, 111-133. MR1317629 (96b:78035)

[21] M. Mitrea and M. Muether, An integration by parts formula in submanifolds of positive codimension, Math. Methods Appl. Sci., Vol. 27, No.4 (2004), 1711-1723. MR2089158 (2005i:58008)

[22] J.D. Sykes and R.M. Brown, The mixed boundary problem in $L^{p}$ and Hardy spaces for Laplace's equation on a Lipschitz domain, Harmonic analysis and boundary value problems (Fayetteville, AR, 2000), pp. 1-18, Contemp. Math., 277, Amer. Math. Soc., Providence, RI, 2001. MR1840423 (2002g:35058)

[23] M. Taylor, Partial Differential Equations, Vol. I-III, Applied Mathematical Sciences, 116, Springer-Verlag, New York, 1996. MR1395149 (98b:35003)

Department of Mathematics, University of Missouri at Columbia, Columbia, Missouri 65211

E-mail address: mitream@missouri.edu 